\title{
Deciphering the Algebraic CPT Theorem
}

\author{
Noel Swanson*
}

October 17, 2018

\begin{abstract}
The CPT theorem states that any causal, Lorentz-invariant, thermodynamically well-behaved quantum field theory must also be invariant under a reflection symmetry that reverses the direction of time $(\mathrm{T})$, flips spatial parity $(\mathrm{P})$, and conjugates charge $(\mathrm{C})$. Although its physical basis remains obscure, CPT symmetry appears to be necessary in order to unify quantum mechanics with relativity. This paper attempts to decipher the physical reasoning behind proofs of the CPT theorem in algebraic quantum field theory. Ultimately, CPT symmetry is linked to a systematic reversal of the $C^{*}$-algebraic Lie product that encodes the generating relationship between observables and symmetries. In any physically reasonable relativistic quantum field theory it is always possible to systematically reverse this generating relationship while preserving the dynamics, spectra, and localization properties of physical systems. Rather than the product of three separate reflections, CPT symmetry is revealed to be a single global reflection of the theory's state space.
\end{abstract}

\section{Contents}

1 Introduction: The CPT Puzzle 2

2 The Algebraic CPT Theorem 5

2.1 Assumptions . . . . . . . . . . . . . . . . . . . 7

2.2 Charges and Superselection Structure . . . . . . . . . . . . . 9

2.3 CPT Symmetry and the Bisognano-Wichmann Property . . . . . . 11

*Department of Philosophy, University of Delaware, 24 Kent Way, Newark, DE 19716, USA, nswanson@udel.edu 
3 Deciphering the Theorem 14

3.1 The Canonical Involution . . . . . . . . . . . . . . . . . . 15

3.2 The Dual Lie-Jordan Product . . . . . . . . . . . . . . . . . . . . 17

3.3 Tomita-Takesaki Modular Theory . . . . . . . . . . . . . . . . . . 19

3.4 Time Reversal . . . . . . . . . . . . . . . . . . . . . . . . . . . 22

3.5 Wedge Reflection . . . . . . . . . . . . . . . . . . . 24

3.6 Charge Conjugation . . . . . . . . . . . . . . . . . . . 28

3.7 Summary . . . . . . . . . . . . . . . . . . . . 32

4 Discussion 33

4.1 Explaining CPT Invariance . . . . . . . . . . . . . . . 33

4.2 Antimatter and Time Reversal . . . . . . . . . . . . . . . 37

4.3 Greaves's Structural Explanation . . . . . . . . . . . . . . . 39

4.4 CPT Symmetry and state space Orientation . . . . . . . . . . 43

4.5 Classical or Quantum? . . . . . . . . . . . . . . . 46

\section{Introduction: The CPT Puzzle}

Virtually every serious candidate for a fundamental physical theory from Newtonian gravitation to classical electrodynamics has been time reversal invariant for every nomologically possible world, there is an otherwise identical nomologically possible world where the direction of time is reversed. Surprisingly, this is not true for relativistic quantum field theories (QFTs). It is possible to write down physically reasonable QFTs which are not time reversal invariant, and as James Cronin and Val Fitch demonstrated in 1964, weak nuclear interactions in the actual world are described by such a theory. ${ }^{1}$

While QFTs may fail to be symmetric under simple time reversal, a deep foundational theorem ensures that there is always a more complicated time reversal symmetry present. The CPT theorem states that any causal, Lorentz invariant, thermodynamically well-behaved QFT must be invariant under a combined symmetry operation that reverses the direction of time $(\mathrm{T})$, flips spatial parity $(\mathrm{P})$, and conjugates all charges present in the theory $(\mathrm{C})$. Since particles and antiparticles carry opposite charge, the net effect of charge conjugation is to swap matter and antimatter. In a CPT invariant theory, every nomologically possible world has a

\footnotetext{
${ }^{1}$ In work that would win them the 1980 Nobel Prize, Cronin and Fitch observed that neutral kaons transform into their antiparticle partners at a different rate than the reverse process.
} 
dopplegänger where the future is the past, right is left, and you and I are made out of antiparticles. ${ }^{2}$

It is far from obvious why this should be the case. Why is this seemingly ad hoc combination of reflections always a symmetry of nature? Greaves (2010) observes that the theorem becomes even more puzzling when one recasts it in terms of possible temporal, spatial, and charge orientation structures that the laws of nature might make use of. If a theory fails to be time reversal invariant, it does so because its laws somehow pick out a distinguished temporal direction. The CPT theorem then says that no well-behaved QFT can make use of one kind of orientation independently of the other two kinds. The laws can single out a preferred temporal orientation, but only up to a choice of spatial handedness and charge sign. The puzzle, as Greaves notes, is that these orientation structures appear to be "paradigm cases of distinct existences; it's odd to find such necessary connections between them" (2010, p. 38). Indeed, in relativistic spacetime one can show that spatial orientation and temporal orientation are mathematically independent; a choice of one does not fix the other. While their relationship to charge orientation is less clear cut, charge superselection structure arises from internal gauge symmetries associated with the particular forces that the charges couple to, and these internal symmetries have no direct connection to spacetime structure. ${ }^{3}$

Such a connection appears essential, however, in order to unify quantum mechanics and special relativity. Straightforward extensions of Schrödinger wave mechanics to relativistic spacetime generate non-physical negative energy states. A series of no-go results show that it is impossible to excise these states while maintaining locality and Lorentz invariance (Strocchi, 2013, ch. 1). Interestingly, in quantum field theory it becomes possible to reinterpret negative energy states as positive energy antiparticle states. This trick only works to restore Lorentz invariance, though, if there is an exact correspondence between particles and antiparticles; they must be indistinguishable except for their charge. The CPT theorem accounts for this, explaining why particle/antiparticle pairs have the same mass,

\footnotetext{
${ }^{2} \mathrm{As}$ in the case of $\mathrm{T}$ invariance, $\mathrm{CPT}$ invariance is often interpreted as indicating that these apparently distinct possibilities are in fact different representations of the same physically possible world. For present purposes, I will set aside this interpretive question.

${ }^{3}$ Here the Coleman-Mandula theorem (Coleman and Mandula, 1967) gives us prima facie support for a kind of independence result. The theorem says that with the exception of supersymmetry, there is no non-trivial way to combine internal gauge symmetries and external spacetime symmetries of the QFT scattering matrix. This result makes a necessary connection between $\mathrm{C}$ and $\mathrm{PT}$ symmetries all the more puzzling.
} 
spin, and lifetime. It is therefore a result with deep foundational significance.

Despite its importance, the physical basis for CPT symmetry remains obscure. As Bain (2016) emphasizes, part of the problem is that there are several different versions of the theorem with different starting assumptions. Many of the more technical assumptions do not have a clear physical interpretation, making comparisons between various proofs challenging. To compound this difficulty, the theorems are couched within different mathematical frameworks, Lagrangian QFT, Wightman QFT, S-matrix QFT, and algebraic QFT. The Lagrangian and S-matrix proofs, while more physically transparent, lack mathematical rigor, whereas the rigorous axiomatic proofs in the Wightman and algebraic frameworks are more physically opaque. This state of affairs has prompted Greaves and Thomas (2014) to formulate a rigorous Lagrangian version of the CPT theorem based on central ideas from Bell (1955). In this paper I attack the problem from the opposite direction, by looking for a more physically perspicuous interpretation of proofs in algebraic QFT, the framework most familiar to philosophers of physics. ${ }^{4}$

In $\S 2$, I give an overview of algebraic QFT, focusing on the features most essential for understanding the CPT theorem. Extant algebraic proofs rely on the following main idea: in the vacuum representation of any physically well-behaved QFT, certain local algebraic invariants associated with spacelike wedge regions act geometrically as elements of the Poincaré group. One of these invariants, the modular conjugation operator, $J_{W}$, implements a full CPT transformation of the theory when combined with a spatial rotation. But why do modular invariants play such a pivotal role, and what makes wedge regions so special?

The central portion of the paper, $\S 3$, takes the form of a mathematical physics whodunit. If we suspect that a generic QFT must have some generalized timereversal symmetry, where might we look for it in the structure of algebraic QFT? As we dissect the theorem piece by piece, we will discover why the wedge modular conjugation must be the culprit.

\footnotetext{
${ }^{4}$ This paper is based on previous dissertation work (Swanson, 2014, ch. 3). The main conclusions drawn and the broad structural account of the algebraic CPT theorem are the same, but some of the central details are different. In particular, the distinction between *-isomorphisms, anti-isomorphisms, and conjugate-isomorphisms are more clearly drawn by lemma 1 and directly connected to modular theory by lemma 2 . Rather than starting from modular covariance, the present account uses Borchers's auxiliary analyticity assumptions to more clearly link central steps in the proof back to the Haag-Kastler axioms, thereby reinforcing the arguments in Swanson (2018). The discussion of charge conjugation in $\S 3.6$ is also different, highlighting the importance of modular inclusions and hewing more closely to existing proofs in the mathematical physics literature. The natural interdefinability of temporal, spatial, charge, and state space orientations will be explored in more detail in future work.
} 
Our detective work points towards an intriguing resolution of the CPT puzzle. In QFT, temporal, spatial, and charge orientation structures are less independent than they look. As we explore the structural explanation supplied by the algebraic CPT theorem in $\S 4$, we will see that the reflection implemented by $J_{W}$ is not the product of three separate operations at all, but rather a single, global state space reflection which systematically reverses the connection between symmetries and the conserved quantities that generate them. Whereas Greaves (2010) maintains that the theorem is essentially a relativistic result, the present discussion suggests that the theorem relies on central assumptions from both relativity and quantum mechanics.

\section{The Algebraic CPT Theorem}

One of the most mathematically rigorous approaches to QFT currently on the table, algebraic QFT (AQFT) serves as a natural framework for investigating the conceptual underpinnings of relativistic quantum theories. Rather than beginning with the specification of a Hilbert space, AQFT starts with an abstract characterization of the algebraic properties of gauge-invariant physical quantities known as observables. It is typically assumed that the observables of a quantum system form the self-adjoint part of a noncommutative $C^{*}$-algebra. Such an algebra, $\mathfrak{A}$, is a linear vector space over $\mathbb{C}$ equipped with a noncommutative multiplication operation and closed under a canonical involution mapping, ${ }^{*}: \mathfrak{A} \rightarrow \mathfrak{A}$, satisfying:

$$
\begin{aligned}
& \left(A^{*}\right)^{*}=A, \quad(A+B)^{*}=A^{*}+B^{*} \\
& (c A)^{*}=\bar{c} A^{*}, \quad(A B)^{*}=B^{*} A^{*},
\end{aligned}
$$

where $c \in \mathbb{C}$, and $\bar{c}$ denotes complex conjugation. Additionally, $\mathfrak{A}$ must be complete with respect to the uniform topology induced by a norm, $\|$ II, subject to the constraints,

$$
\left\|A^{*} A\right\|=\|A\|^{2}, \quad\|A B\| \leq\|A\|\|B\| .
$$

In what follows, the involution mapping plays a major role. It is the abstract analogue of the Hilbert space adjoint operation, and allows us to define important subsets of elements. An operator is self-adjoint if $A=A^{*}$, an isometry if $A^{*} A=I$, unitary if $A A^{*}=A^{*} A=I$, and a projection if $A A^{*}=A$. A Von Neumann algebra is a $C^{*}$-algebra that contains a complete lattice of projection operators. Such algebras can be classified into different types based on the structure of this lattice. Finite algebras, commonly used in nonrelativistic quantum mechanics, are always 
isomorphic to the algebra of bounded operators on a finite dimensional Hilbert space. Infinite algebras, employed in QFT and quantum statistical mechanics, are isomorphic to a subalgebra of bounded operators on an infinite dimensional Hilbert space. As we will see in $\S 3$, the proof of the CPT theorem hinges on the physical interpretation of the involution mapping and its implementation by modular invariants of infinite von Neumann algebras.

A state, $\phi: \mathfrak{A} \rightarrow \mathbb{C}$, is a (normalized, positive) linear functional whose values represent the expectation values of observables in $\mathfrak{A}$. Given a state, the GelfandNaimark-Segal (GNS) construction determines a unique representation, $\pi_{\phi}(\mathfrak{A})$, of $\mathfrak{A}$ as a concrete subalgebra of bounded linear operators acting on a separable Hilbert space, $\mathcal{H}_{\phi}$. Within a representation, the closure of $\pi_{\phi}(\mathfrak{A})$ with respect to the Hilbert space weak topology defines a von Neumann algebra equivalent to the double commutant, $\pi_{\phi}(\mathfrak{A})^{\prime \prime}$. In AQFT, this procedure allows for the definition of additional representation-dependent observables including the energy-momentum operators and superselected charges. Two representations $\pi_{\phi_{1}}, \pi_{\phi_{2}}$ are quasiequivalent iff $\pi_{\phi_{1}}(\mathfrak{A})^{\prime \prime}$ and $\pi_{\phi_{2}}(\mathfrak{A})^{\prime \prime}$ are ${ }^{*}$-isomorphic. Because field systems in AQFT have infinitely many degrees of freedom, GNS representations of a given algebra will typically not be quasiequivalent (unlike the situation in non-relativistic quantum mechanics).
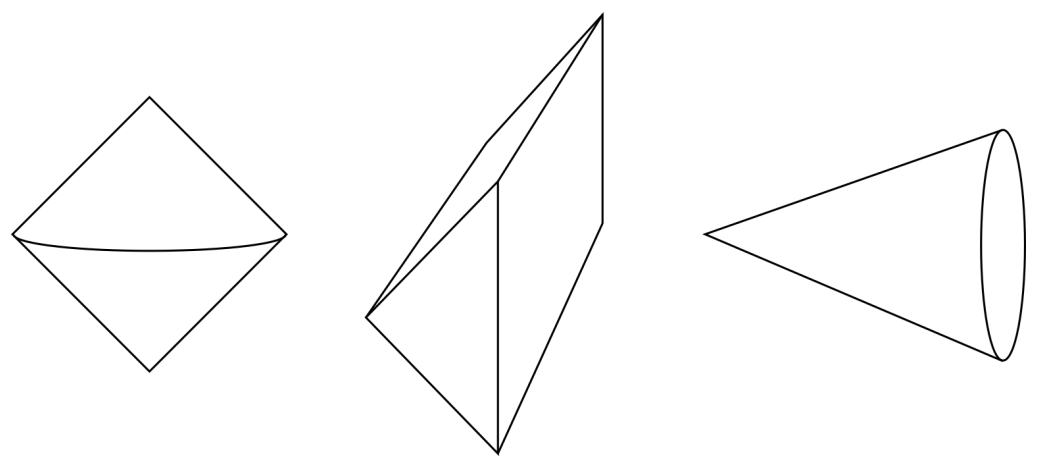

Figure 1: A doublecone, a spacelike wedge, and a spacelike cone.

We will focus on AQFT in flat spacetime. Throughout, $O$ will denote an open region of Minkowski spacetime and $O^{\prime}$ the interior of its causal complement, the set of all points spacelike separated from all points in $O$. Certain special regions will be important to keep track of. A doublecone is a compact region formed by the intersection of a past and future lightcone at two timelike separated points. A spacelike wedge is an infinitely extended wedge-shaped region, whose two defining 
planes are tangent to the edges of some lightcone. A spacelike cone is a cone-shaped subset of a spacelike wedge, infinitely extended in one spacelike direction.

\section{$2.1 \quad$ Assumptions}

With these preliminary definitions in place, a model of AQFT is given by an assignment, $\{\mathfrak{A}(O)\}$, of $C^{*}$-algebras to regions of spacetime satisfying the HaagKastler axioms, along with a set of physically possible states, $\{\phi\}$. Each state in $\{\phi\}$ determines a GNS representation and a corresponding assignment of local von Neumann algebras, $\left\{\mathfrak{R}_{\phi}(O)\right\}=\left\{\pi_{\phi}(\mathfrak{A}(O))^{\prime \prime}\right\}$. The self-adjoint elements of these algebras represent locally measurable physical quantities, while the Haag-Kastler axioms specify the dynamics and enforce the joint requirements of relativity and quantum mechanics. There are five standard axioms, and they all play a crucial role in the algebraic CPT theorem:

Isotony: If $O_{1} \subset O_{2}$, then $\mathfrak{A}\left(O_{1}\right) \subset \mathfrak{A}\left(O_{2}\right)$. This gives the assignment $\{\mathfrak{A}(O)\}$ the structure of a net and allows us to define the quasilocal algebra, $\mathfrak{A}$, as its upwards inductive limit. (The family of physical states $\{\phi\}$ is formally defined as a set of states of $\mathfrak{A}$.)

Microcausality: If $O_{1} \subset O_{2}^{\prime}$, then $\mathfrak{A}\left(O_{1}\right)$ and $\mathfrak{A}\left(O_{2}\right)$ commute. This enforces relativistic no-signaling constraints, ruling out act-outcome correlations at spacelike separation. (It is also sometimes called the Einstein causality or locality axiom.)

Covariance: The net $\{\mathfrak{A}(O)\}$ transforms covariantly under a strongly continuous representation of the connected Poincaré group (or more generally its covering group) as automorphisms of $\mathfrak{A}$. The full group of isometries of Minkowski spacetime is the Poincaré group, while its covering group has the same Lie algebra and is used to represent symmetries of spinor fields. The connected Poincaré group is the subgroup topologically connected to the identity, consisting of translations, rotations, and boosts. (It does not include orientation-reversing isometries like $\mathrm{P}, \mathrm{T}$, or $\mathrm{PT}$ reflections.) The dynamical laws of the theory are encoded in the translation subgroup of this representation and are guaranteed to be Lorentz invariant.

Vacuum: There exists at least one translation invariant state, $\omega \in\{\phi\}$. This is a necessary condition for $\omega$ to be interpretable as a vacuum state. 
In the corresponding GNS representation, the translation subgroup is implemented by a strongly continuous 1-parameter group of unitary operators, $U(a)$. The group generators are the energy-momentum observables and are affiliated with the global von Neumann algebra, $\mathfrak{R}_{\omega}=\pi_{\omega}(\mathfrak{A})^{\prime \prime}$.

Spectrum Condition: In each vacuum GNS representation, the energymomentum observables have spectral support in the same lightcone lobe in momentum space. This ensures that the energy spectrum is bounded from below in all Lorentz frames and that the vacuum is thermodynamically stable. ${ }^{5}$

In addition to the Haag-Kastler axioms, the algebraic CPT theorem relies on five other assumptions:

Additivity For any family of bounded open regions, $\left\{O_{i}\right\}$, the local algebra $\mathfrak{A}\left(\cup O_{i}\right)$ is the $C^{*}$-algebra generated by the family of local algebras $\left\{\mathfrak{A}\left(O_{i}\right)\right\}$. This is a technical condition relating the algebras of bounded and unbounded regions. It is used in the analysis of charge superselection structure, and it entails weak additivity in vacuum representations. Weak additivity ensures that the global von Neumann algebra $\mathfrak{R}_{\omega}$ can be generated by translations of any local algebra $\mathfrak{R}_{\omega}(O)$. It is an important ingredient in the Reeh-Schlieder theorem and several crucial lemmas in the algebraic CPT theorem.

Wedge Intersection Property: For any doublecone D, in any vacuum representation, $\mathfrak{R}_{\omega}(D)=\bigcap \mathfrak{R}_{\omega}\left(W_{i}\right)$ for all spacelike wedges $W_{i} \supset D$. This is another technical condition allowing vacuum doublecone algebras to be defined by the intersection of families of wedge algebras. It is used to in the proof of the Bisognano-Wichmann property and to construct the minimal Poincaré representation in $§ 3.5$. If a model of AQFT does not satisfy the wedge intersection property it is always possible to expand the net of local algebras so that it is satisfied, although the extension will not typically be unique.

Split Property: If regions $\mathrm{O}_{1}$ and $\mathrm{O}_{2}$ are spacelike separated and not tangent, then in the vacuum representation $\mathfrak{R}_{\omega}\left(O_{1}\right)$ and $\mathfrak{R}_{\omega}\left(\mathrm{O}_{2}\right)$ can be "split,"

\footnotetext{
${ }^{5}$ As usually formulated, the spectrum condition requires that the spectral support of $U(a)$ lie in the closed forward lightcone, $\overline{V^{+}}$, in momentum space. The apparent reference to a distinguished temporal orientation is eliminable. It is only required that $U(a)$ must have spectral support in a closed convex set $\bar{V}$ which is asymmetric under taking additive inverses: $\{\bar{V}\} \cap\{-\bar{V}\}=\{0\}$.
} 
i.e., they generate a tensor product of von Neumann algebras. Along with the spectrum condition, the split property is part of the characterization of thermodynamically well-behaved QFTs. It entails that the family $\{\phi\}$ includes well-defined thermal equilibrium states satisfying the Kubo-MartinSchwinger (KMS) condition and is a necessary condition for a model of AQFT to have an emergent particle interpretation (Haag, 1996, ch. V.5). Existing algebraic proofs of the CPT theorem rely on the weaker distal split property, which only requires the existence of some pair of spacelike separated wedges such that $\mathfrak{R}_{\omega}\left(W_{1}\right)$ and $\mathfrak{R}_{\omega}\left(W_{2}\right)$ can be split.

Analyticity: At certain critical stages, proofs of the algebraic CPT theorem, like proofs in the Wightman framework, rely on tricky analytic continuation arguments. As we will go on to see, in AQFT many important analyticity properties are derived from the Haag-Kastler axioms and weak additivity. It remains an open question if these assumptions along with the split property are sufficient to derive all of the analyticity needed for the CPT theorem. Existing algebraic proofs require auxiliary analyticity assumptions, and the choice of which assumptions to make marks a place where different algebraic proofs diverge. In our presentation, two closely related assumptions, $B$ analyticity and B-reality, will be introduced in $§ 3.5-6$ once we have developed the necessary technical machinery.

DHR/BF Selection Criteria: For every physical state $\phi \in\{\phi\}$, the GNS representation $\pi_{\phi}(\mathfrak{A})^{\prime \prime}$ is quasiequivalent to the vacuum representation in the causal complement of some doublecone or spacelike cone. This final assumption is the key to the algebraic analysis of charge structure. Its physical motivation is the subject of the next section.

\subsection{Charges and Superselection Structure}

Rather than a single Hilbert space, the state space of a model of AQFT is a collection of different GNS representations, grouped into unitary equivalence classes called sectors. Each representation in a given sector has the same folium of density operators, representing states with the same global boundary conditions, characterized by the values of representation-dependent observables in the global algebra $\mathfrak{R}_{\phi}=\pi_{\phi}(\mathfrak{A})^{\prime \prime}$. In different models of AQFT, different families of global states and their corresponding GNS representations carry physical significance. The analysis of charge representations initiated by Doplicher, Haag, and Roberts (1969a,b) is 
one of the crowning achievements of AQFT and plays a central role in the algebraic CPT theorem.

Charges are gauge invariant, conserved quantities associated with particular force laws. For example, electric charge is the conserved quantity that couples to the electromagnetic force; color charge is the conserved quantity that couples to the strong force. ${ }^{6}$ Besides satisfying global conservation laws, they obey superselection rules that forbid states which are superpositions of different charges. In addition, charges can be localized within some region of spacetime, and every charge has a well-defined conjugate charge. Particles carrying conjugate charges can annihilate each other, producing pure energy. Conversely, particle/antiparticle pairs can spontaneously spring from the vacuum state.

In AQFT, these features are captured using special mappings of the quasilocal algebra into itself called localized transportable endomorphisms. Formally, such a morphism is an injective ${ }^{*}$-homomorphism, $\varrho: \mathfrak{A} \rightarrow \mathfrak{A}$. It must be localized in some region, $O$ (i.e., it acts as the identity on $\mathfrak{A}\left(O^{\prime}\right)$ ), and it must be possible to transport $\varrho$ to any other similarly shaped region in spacetime using unitary mappings (i.e., for any similar region, $\mathrm{O}_{2}$, there is a localized endomorphism, $\varrho_{2}$, and a unitary operator, $U$, such that $U \varrho(A)=\varrho_{2}(A) U$ for all $\left.A \in \mathfrak{A}\right)$.

The collection of localized transportable endomorphisms has a rich mathematical structure, that of a symmetric tensor *-category. In particular, the category has a natural tensor product which allows us to define notions of charge composition and conjugate charges. Each endomorphism induces a corresponding mapping on global states over $\mathfrak{A}$. If $\omega$ is a vacuum state, $\omega \circ \varrho$ describes a state with charge $Q$ localized in region $O$. Its conjugate is defined as the unique endomorphism, $\bar{\varrho}$, such that $\omega \circ \varrho \circ \varrho$ is a mixed state containing a component in the vacuum sector. This captures the necessary condition for pair creation/annihilation. ${ }^{7}$

Doplicher, Haag, and Roberts analyze charges described by endomorphisms localized in compact spatiotemporal regions. Such charges couple to forces like the strong force, whose strength falls off sharply as a function of distance. They prove

\footnotetext{
${ }^{6}$ The notion of color charge discussed here is not the same as the more familiar quark color labels red, blue, and green. These labels do not have a gauge invariant meaning and can be superimposed. Color charge is a $\mathbb{Z}_{3}$-valued gauge-invariant superelected quantity in the center of $\mathrm{SU}(3)$ constructed from functions of local Casimir invariants. See Kijowski and Rudolph (2003) for a discussion of the superselection structure of quantum chromodynamics on a fnite lattice.

${ }^{7} \mathrm{An}$ example of a common annihilation event is $e^{+}+e^{-} \rightarrow \gamma+\gamma$. Since charge is globally conserved and photons are chargeless, any interaction of this kind requires that particles and antiparticles have conjugate charge. If $\omega \circ \varrho \circ \varrho$ is a mixed state with a component in the vacuum sector, then the probability of a creation/annihilation event is nonzero according to the Born rule.
} 
that the relevant category of localized transportable endomorphisms is equivalent to the category of GNS representations of states satisfying the DHR selection criterion - $\phi$ satisfies the DHR selection criterion if its GNS representation is quasiequivalent to the vacuum representation in the causal complement of some doublecone. The corresponding charge sectors are labeled by the value of the total charge observable, and conjugate sectors are defined by the condition that $\pi(\mathfrak{A})^{\prime \prime} \otimes \bar{\pi}(\mathfrak{A})^{\prime \prime}$ contains a copy of the vacuum representation $\pi_{\omega}(\mathfrak{A})^{\prime \prime}$. According to the DHR picture, matter and antimatter states are represented by states in conjugate sectors, giving rigorous mathematical content to the idea that such states have opposite charge quantum numbers (Baker and Halvorson, 2010).

For theories with compactly localized charges like quantum chromodynamics, the DHR selection criterion is a physically plausible constraint on the family of possible global states $\{\phi\}$. Buchholz and Fredenhagen (1982) extend the DHR picture to include topological charges localized in spacelike cones. In theories with a mass gap, there is a 1-1 correspondence between particle representations and states satisfying the BF selection criterion - $\phi$ satisfies the BF selection criterion if its GNS representation is quasiequivalent to the vacuum representation in the causal complement of some spacelike cone. While impressive, the analysis of charge structure in AQFT is still incomplete. Because of the infrared problem, we currently lack a full understanding of the localization properties of massless charged particles, and thus the algebraic proof of the CPT theorem cannot be applied to theories like quantum electrodynamics at this stage.

\subsection{CPT Symmetry and the Bisognano-Wichmann Prop- erty}

Within the setting of the DHR/BF picture, we can frame necessary and sufficient conditions for the existence of a CPT symmetry operator, $\Theta$ :

(a) $\Theta$ is unitary or antiunitary, ${ }^{8}$ and if $O_{1} \subset O_{2}$, then $\Theta \mathfrak{A}\left(O_{1}\right) \Theta \subset \Theta \mathfrak{A}\left(O_{2}\right) \Theta$.

(b) $\Theta \mathfrak{A}(O) \Theta=\mathfrak{A}(-O)$, where $-O$ is the region obtained from $O$ via a full spatiotemporal reflection in both the space and time coordinates.

(c) In any representation carrying a unitary representation, $U(a, \Lambda)$, of the connected Poincaré transformations, $\Theta U(a, \Lambda) \Theta=U(-a, \Lambda)$.

\footnotetext{
${ }^{8} \mathrm{An}$ antiunitary operator acting on a Hilbert space must satisfy $V^{*} V=V V^{*}=I$ like a unitary operator, however it is complex antilinear, $V\left(c_{1} \Phi+c_{2} \Psi\right)=\bar{c}_{1} V \Phi+\bar{c}_{2} V \Psi$, rather than linear.
} 
(d) For any charge endomorphism, $\theta \circ \varrho \circ \theta=\bar{\varrho}$, where $\theta$ is the (anti-)isomorphism defined by the adjoint action of $\Theta, \theta(A):=\Theta A \Theta$ for all $A \in \mathfrak{A}$.

The first condition is a general constraint on symmetries in AQFT. Wigner's theorem requires that symmetries must be unitarily or antiunitarily implemented in order to preserve transition probabilities. Additionally, symmetries should preserve the localization information captured by isotony. Conditions (b)-(c) ensure that $\Theta$ implements a full spatiotemporal reflection and has the right commutation relations with the Poincaré transformations. In particular, (c) ensures that $\Theta$ commutes with the dynamics and preserves the mass and spin properties of particles. The final condition tells us that $\Theta$ conjugates all charges present in the theory.

This leads to a general statement of the main theorem:

CPT Theorem. Given a model of AQFT satisfying the Haag-Kastler axioms, additivity, the wedge intersection property, the distal split property, and the $D H R / B F$ selection criterion, if the model also satisfies analyticity conditions sufficient to entail the Bisognano-Wichmann property, then there exists an antiunitary CPT operator, $\Theta$, satisfying (a)-(d).

Algebraic proofs of the CPT theorem are based on generalizations of a lesser known result from constructive QFT. Bisognano and Wichmann $(1975,1976)$ prove that for QFTs satisfying the Wightman axioms, local algebras associated with spacelike wedges in the vacuum representation contain special invariants that generate particular Poincaré transformations. The modular unitarites, $\Delta_{W}^{i t}$, associated with $\mathfrak{R}_{\omega}(W)$ generate the unique 1-parameter group of $W$-preserving Lorentz boosts, $\Lambda_{W}(t) .{ }^{9}$ The modular conjugation, $J_{W}$, implements a $\mathrm{P}_{1} \mathrm{~T}$ reflection that reverses the direction of time and flips one spatial direction perpendicular to the edge of the wedge. Interestingly, it turns out that $J_{W}$ also conjugates charge. The CPT theorem is then an immediate corollary of rotational covariance.

\footnotetext{
${ }^{9} \mathrm{~A}$ spacelike wedge is the region of Minkowski spacetime causally connected to an immortal, uniformly accelerating observer, the so-called Rindler wedge. If the observer is accelerating in the $x_{1}$ direction, their trajectory can be written in standard coordinates as

$$
\begin{aligned}
& x^{0}(\tau)=a^{-1} \sinh (\tau) \\
& x^{1}(\tau)=a^{-1} \cosh (\tau) \\
& x^{2}(\tau)=x^{3}(\tau)=0,
\end{aligned}
$$

where $\tau$ is proper time. The wedge region is defined by the condition $x^{1}>\left|x^{0}\right|$. The BisognanoWichmann theorem tells us that in the vacuum representation, $\Delta^{i t}=e^{2 \pi i t K_{1}}$ (where $K_{1}$ is the generator of an $x_{1}$-boost). This is a simple rescaling of proper time translations along the observer's worldline.
} 

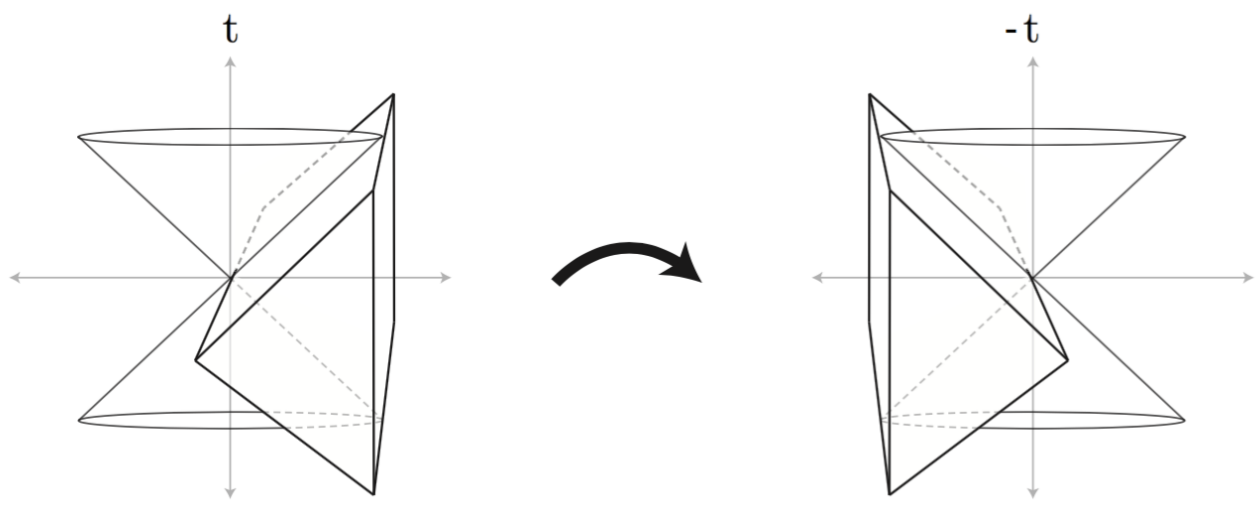

Figure 2: The $\mathrm{P}_{1} \mathrm{~T}$ reflection implemented by $J_{W}$

The original proof of the Bisognano-Wichmann theorem uses extensive analytic continuation techniques relying on the special properties of gauge-dependent Wightman field operators. It thus does not directly apply to AQFT. Nonetheless, mathematical physicists have long suspected that the theorem is actually a more general consequence of the structure of gauge-independent local observables. As we will see in $§ 3.3$, the existence of local modular invariants is a consequence of the Haag-Kastler axioms and weak additivity, and their geometric interpretation is tightly constrained. This motivates the following:

Bisognano-Wichmann Property: In the vacuum representation, for any spacelike wedge $W$, the wedge modular unitaries generate $\Lambda_{W}(t)$.

The Bisognano-Wichmann property holds iff $J_{W}$ implements a $\mathrm{CP}_{1} \mathrm{~T}$ reflection. Algebraic proofs of the CPT theorem therefore attempt to isolate analyticity assumptions that are sufficient for establishing the Bisognano-Wichmann property.

In 2-dimensional theories, no additional assumptions are needed. The first algebraic proof of the CPT theorem, Borchers (1992), inventively uses the analyticity properties entailed by covariance and the spectrum condition to establish the Bisognano-Wichmann property for 2-dimensional models of AQFT. In higher dimensions the situation is less clear. Haag (1996) conjectures that the HaagKastler axioms and the split property should be sufficient to entail the BisognanoWichmann property on their own, but this problem remains unsolved. Although there are models of the Haag-Kastler axioms in which the Bisognano-Wichmann property fails (Yngvason, 1994; Buchholz et al., 2000), there are none that also satisfy the split property. 
The first proof for 3- and 4-dimensional theories, Guido and Longo (1995), drops the covariance axiom and spectrum condition in favor of a geometric constraint on $\Delta_{W}^{i t}$ :

Modular Covariance: In the vacuum representation, for any spacelike wedge $W$,

$$
\Delta_{W}^{i t} \Re_{\omega}(O) \Delta_{W}^{-i t}=\mathfrak{R}_{\omega}\left(\Lambda_{W}(t) O\right)
$$

This requires that the adjoint action of the modular unitaries maps arbitrary local algebras in the vacuum representation onto the algebras of $\Lambda_{W}(t)$-boosted regions. This covariance entails the Bisognano-Wichmann property, but showing that each $\Delta_{W}^{i t}$ acts geometrically as a boost requires a detailed argument exploiting the algebraic and analytic properties of the modular invariants. Bain (2016) draws a number of philosophical conclusions about the algebraic CPT theorem (e.g., that it does not assume Lorentz invariance) based on a direct reading of the GuidoLongo proof. Swanson (2018) cautions against such a direct reading, arguing that modular covariance essentially bundles together the covariance axiom, spectrum condition, and additional analyticity properties, obscuring the physical justification behind various steps in the proof.

Here I will focus on the approach of Borchers (1995, 1996a, 1998, 2000), which seeks to identify precisely which analyticity conditions are needed in higher dimensions in addition to those already implicitly encoded in the Haag-Kastler axioms. Although in their present form these conditions are quite technical and their physical motivation is poorly understood, pursuing this strategy will enable us to trace the clearest possible chain of argument back to the Haag-Kastler axioms. ${ }^{10}$

\section{Deciphering the Theorem}

Summing up the mathematical philosophy behind AQFT, Halvorson and Müger (2006, p. 740) observe:

AQFT proceeds by isolating some structural assumptions that hold in most known QFT models. It formalizes these structural assumptions,

\footnotetext{
${ }^{10}$ There are several other approaches that deserve mention. Kuckert (1997) proves that if the wedge modular invariants map open regions onto open regions, then the Bisognano-Wichmann property follows. Buchholz et al. (2000) employs an alternative geometric constraint, the condition of geometric modular action, on the family of wedge modular conjugations $\left\{J_{W}\right\}$. Mund (2001) proves the Bisognano-Wichmann property for asymptotically complete QFTs with a mass gap using elementary algebraic assumptions and tools from Haag-Ruelle scattering theory.
} 
and then uses "abstract but efficient nonsense" to derive consequences of these assumptions.

Prima facie, the algebraic CPT theorem is a paradigm example of this approach. With the exception of technical conditions like additivity, the wedge intersection property, and analyticity, its main structural inputs are reasonably physically transparent. The chain of argument is anything but. Somehow, using the geometric properties of wedge-localized modular invariants, we can construct an extended representation of the Poincaré group which miraculously includes an antiunitary CPT operator. To make the situation even more challenging, presentations of the CPT theorem in the mathematical physics literature typically start at a very late stage of the argument, are spread over many separate papers, and often appeal to more abstract assumptions than the Haag-Kastler axioms, aiming for the highest level of mathematical generality possible. (Frequently they seek to prove the spin-statistics theorem at the same time.) The goal of this section is to decipher the chain of physical reasoning behind the CPT theorem, starting from a more elementary algebraic foundation and proceeding slowly and carefully through the core of the proof. The argument is certainly abstract, but it is less nonsensical than it first appears.

\subsection{The Canonical Involution}

We suspect that a generic model of AQFT should contain a hidden CPT reflection symmetry, but the Haag-Kastler axioms only require covariance with respect to connected Poincaré symmetries. How can a reflection like CPT get into the mix?

Our first important observation is that there is already an algebraic reflection symmetry hiding in plain sight - in every $C^{*}$-algebra, the canonical involution can be viewed as a reflection of the algebra across its self-adjoint subspace. Every operator in $\mathfrak{A}$ can be uniquely written in "complex form," $A=H+i K$, where $H=\frac{1}{2}\left(A+A^{*}\right)$ and $K=\frac{i}{2}\left(A^{*}-A\right)$ are self adjoint. A quick calculation reveals that the canonical involution mapping, * $: \mathfrak{A} \rightarrow \mathfrak{A}$, acts as "complex conjugation," sending $A=H+i K$ to $A^{*}=H-i K$ and leaving the self-adjoint subspace, $\mathfrak{A}_{S A}$, pointwise invariant. Thus just like the complex numbers, a $C^{*}$-algebra is selfsimilar; there is a conjugation operation that reflects the algebra across its "real axis," $\mathfrak{A}_{S A}$. The positive and negative "imaginary halves" of the algebra, while distinct, have identical algebraic properties.

Strictly speaking, the canonical involution is not an automorphism of $\mathfrak{A}$. It reverses the order of operator multiplication, $(A B)^{*}=B^{*} A^{*}$, and it is conjugate- 
linear on the underlying vector space, $\left(c_{1} A+c_{2} B\right)^{*}=\overline{c_{1}} A^{*}+\overline{c_{2}} B^{*}$. It restricts to the identity on $\mathfrak{A}_{S A}$, however, and since physical quantities are represented by self-adjoint operators, this suggests that we should interpret it as a symmetry. Moreover, while the order of multiplication and the difference between $i$ and $-i$ matters within the algebra, from the outside looking in, the choice of a operator product and complex unit looks like an arbitrary convention. We could choose right instead of left operator multiplication and $-i$ rather than $i$ as a complex unit and still be able to encode the same algebraic relations.

We can capture this intuition as follows. Let $\mathfrak{A}^{o p}$ denote the opposite algebra relative to $\mathfrak{A}$, consisting of the same underlying vector space, involution, and norm as $\mathfrak{A}$, but with the opposite $C^{*}$-product, $(A B)^{o p}=B A$. Similarly, let $\mathfrak{A}^{c}$ denote the conjugate algebra, consisting of the same involution, norm, and operator product as $\mathfrak{A}$, but whose underlying vector space is conjugate, $i^{c}=-i$. Finally, let $\mathfrak{A}^{c o p}$ denote the analogously defined conjugate-opposite algebra.

We say that two $C^{*}$-algebras are ${ }^{*}$-isomorphic if there exists a linear bijection between them that preserves the identity, involution, and the operator product. (These conditions entail that the norm is also preserved.) An anti-isomorphism is similarly defined but reverses the order of the operator product, while a conjugateisomorphism acts conjugate linear on the underlying vector space. A conjugateanti-isomorphism does both. It follows from this cluster of definitions that $\mathfrak{A}$ is antiautomorphic, conjugate-automorphic, or conjugate-antiautomorphic (to itself) iff $\mathfrak{A}$ is isomorphic to $\mathfrak{A}^{o p}, \mathfrak{A}^{c}$, or $\mathfrak{A}^{c o p}$ respectively.

Lemma 1. Let $\mathfrak{A}$ be any $C^{*}$-algebra:

(i) $\mathfrak{A}$ is naturally isomorphic to $\mathfrak{A}^{c o p}$,

(ii) $\mathfrak{A}^{o p}$ is naturally isomorphic to $\mathfrak{A}^{c}$,

(iii) $\mathfrak{A}$ is naturally anti-isomorphic to $\mathfrak{A}^{\text {op }}$ and $\mathfrak{A}^{c}$,

(iv) $\mathfrak{A}$ is naturally conjugate-isomorphic to $\mathfrak{A}^{\text {op }}$ and $\mathfrak{A}^{c}$,

with the relevant isomorphisms defined by the involution structure common to all four algebras. ${ }^{11}$

\footnotetext{
${ }^{11}$ Proof. All four algebras have the same involution structure and self-adjoint subspace (which includes the identity element). To prove (i), define the isomorphism $\varphi: \mathfrak{A} \rightarrow \mathfrak{A}^{\text {cop }}$ as the linear mapping whose restriction to $\mathfrak{A}_{S A}$ is the identity and that sends $i \mapsto i^{c}$ and $A B \mapsto(A B)^{o p}$. It is well-defined because the product of two self-adjoint elements is self adjoint iff they commute (and thus iff $A B=(A B)^{o p}$ ), and $i A$ is not self-adjoint for any self-adjoint element $A$. Using
} 
This lemma precisely characterizes the sense in which a $C^{*}$-algebra is self-similar: $\mathfrak{A}$ is naturally conjugate-antiautomorphic to itself, with the canonical involution defining the relevant reflection symmetry. Furthermore, it reveals that there is an entire family of related isomorphisms linking $C^{*}$-algebras with opposite choices of operator product and complex unit.

\subsection{The Dual Lie-Jordan Product}

Do these formal algebraic symmetries have any physical consequences? If the observables are all contained in $\mathfrak{A}_{S A}$, what role does the "imaginary" part of the algebra play? Our second important observation, following Alfsen and Shultz (2001, 2003), is that observables have dual roles - they represent physical quantities and they act as infinitesimal generators of symmetries. The $C^{*}$-algebraic product is in fact two products in disguise:

Theorem (Alfsen-Shultz). In any $C^{*}$-algebra, the operator product has a natural decomposition,

$$
A B=A \bullet B-i(A \star B),
$$

where $A \bullet B:=\frac{1}{2}(A B+B A)$ is a commutative, non-associative Jordan product, and $A \star B:=\frac{i}{2}(A B-B A)$ is a noncommutative, associative Lie product. ${ }^{12}$

the fact from the main text, every element $A \in \mathfrak{A}$ can be uniquely written as $A=H+i K$ with $H, K \in \mathfrak{A}_{S A}$. Consequently $\varphi(A)=H+i^{c} K=H-i K=A^{*}$, so the map is a bijection. Moreover, $\varphi(I)=I$ and $\varphi\left(A^{*}\right)=H-i^{c} K=H+i K=A^{* *}=\varphi(A)^{*}$, so it preserves the identity element and involution structure. Finally, it is multiplicative, since $\varphi(A B)=(A B)^{*}=B^{*} A^{*}=$ $\left(A^{*} B^{*}\right)^{o p}=(\varphi(A) \varphi(B))^{o p}$, and is thus a *-isomorphism. Naturality follows from the fact that for any ${ }^{*}$-homomorphism, $\pi, \pi\left(A^{*}\right)=\pi(A)^{*}$, and therefore $\pi \circ \varphi(A)=\pi\left(A^{*}\right)=\pi(A)^{*}=\varphi \circ \pi(A)$.

The proof of (ii) follows the same reasoning, with $\tilde{\varphi}: \mathfrak{A}^{o p} \rightarrow \mathfrak{A}^{c}$ the analogously defined natural ${ }^{*}$-isomorphism. To prove (iii) define the anti-isomorphism $\varphi^{o p}: \mathfrak{A} \rightarrow \mathfrak{A}^{o p}$ as the linear mapping whose restriction to $\mathfrak{A}_{S A}$ is the identity and that sends $i \mapsto i$ and $A B \mapsto(B A)^{o p}$. $\tilde{\varphi} \circ \varphi^{o p}: \mathfrak{A} \rightarrow \mathfrak{A}^{c}$ then defines an anti-isomorphism between $\mathfrak{A}$ and $\mathfrak{A}^{c}$. Similarly, to prove (iv) define the conjugate-isomorphism $\varphi^{c}: \mathfrak{A} \rightarrow \mathfrak{A}^{c}$ as the conjugate-linear mapping whose restriction to $\mathfrak{A}_{S A}$ is the identity and that sends $i \mapsto-i^{c}$ and $A B \mapsto A B$. $\tilde{\varphi}^{-1} \circ \varphi^{c}: \mathfrak{A} \rightarrow \mathfrak{A}^{o p}$ then defines a conjugate-isomorphism between $\mathfrak{A}$ and $\mathfrak{A}^{o p}$. Naturality follows from the naturalness of $\tilde{\varphi}$ and the fact that $\varphi^{o p}(A)=\varphi^{c}(A)=A$, and so both mappings commute with *-homomorphisms.

Interestingly, $\mathfrak{A}$ is not necessarily isomorphic to $\mathfrak{A}^{o p}$ or $\mathfrak{A}^{c}$. The first such examples are due to Connes (1975). It remains an open question which (if any) natural constraints might entail that an algebra is antiautomorphic (equiv. conjugate-automorphic) to itself.

${ }^{12}$ See Alfsen and Shultz (2003, ch. 6). The Jordan product satisfies the Jordan identity,

$$
\left(A^{2} \bullet B\right) \bullet A=A^{2} \bullet(B \bullet A),
$$


The self-adjoint subspace, $\mathfrak{A}_{S A}$, is closed under the Jordan product which encodes all spectral information about the observables. ${ }^{13}$ The Jordan product therefore captures the way in which observables represent physical quantities. The Lie product, on the other hand, captures the way in which observables generate symmetries. Each element $A \in \mathfrak{A}_{S A}$ defines a 1-parameter group of automorphisms of $\mathfrak{A}$, given by

$$
\alpha_{t}(X):=e^{i t A} X e^{-i t A}
$$

for all $t \in \mathbb{R}, X \in \mathfrak{A}$. Infinitesimally, this can be rewritten in terms of the Lie product:

$$
\begin{aligned}
\left.\frac{d \alpha_{t}(X)}{d t}\right|_{t=0} & =i(A B-B A) \\
& =2(A \star X)
\end{aligned}
$$

Thus the Lie product $A \star X$ represents the tangent vector of the flow associated with the group of symmetries defined by $A$ at $t=0$. Unlike the Jordan structure, $\mathfrak{A}_{S A}$ is not closed under the Lie product. In fact the closure of $\mathfrak{A}_{S A}$ with respect to the Lie product is the entire $C^{*}$-algebra. This reveals that the imaginary part of $\mathfrak{A}$ algebraically encodes the generating relationship between observables and symmetries.

Putting this result together with lemma 1, we see that the four algebras we have introduced, $\mathfrak{A}, \mathfrak{A}^{o p}, \mathfrak{A}^{c}$, and $\mathfrak{A}^{c o p}$, all have the same Jordan product, so they agree on spectral properties of observables. The choice of a $C^{*}$-operator product,

where $A^{2}:=A \bullet A$, while the Lie product satisfies the well-known Jacobi identity,

$$
A \star(B \star C)+C \star(A \star B)+B \star(C \star A)=0 .
$$

They also satisfy two important compatibility conditions, the Leibniz rule and the associator identity:

$$
A \star(B \bullet C)=(A \star B) \bullet C+B \bullet(A \star C), \quad \text { and }(A \bullet B) \bullet C-A \bullet(B \bullet C)=(A \star C) \star B .
$$

The first tells us that that the map $B \mapsto A \star B$ is a derivation on $\left(\mathfrak{A}_{S A}, \bullet\right)$ viewed as a real Jordan algebra. The second quantifies the departure from associativity of the Jordan product and is linked to the Heisenberg uncertainty relations. This structure allows us to canonically view the original $C^{*}$-algebra, $\mathfrak{A}$, as a dual Lie-Jordan algebra defined on the complexified space $\mathfrak{A}_{S A}+i \mathfrak{A}_{S A}$. See Zalamea (2018) for an analysis of the physical significance of this dual structure in both quantum and classical mechanics.

${ }^{13}$ The spectrum of $A \in \mathfrak{A}_{S A}$ is defined as the set of real numbers $\lambda$ such that $A-\lambda I$ is not invertible. The invertibility of $A-\lambda I$ is equivalent to the existence of $B \in \mathfrak{A}_{S A}$ such that $(A-\lambda I) \bullet B=I$ and $(A-\lambda I)^{2} \bullet B=(A-\lambda I)$ (Alfsen and Shultz, 2003, Lem. 1.16-Cor. 1.19). 
Lie product, and complex unit are constrained — specifying any two naturally defines a choice for the third:

\begin{tabular}{c|c|c|c} 
Algebra & $C^{*}$-Product & Lie Product & Complex Unit \\
\hline $\mathfrak{A}$ & $A B$ & $A \star B$ & $i$ \\
$\mathfrak{A}^{\text {op }}$ & $B A$ & $B \star A$ & $i$ \\
$\mathfrak{A}^{c}$ & $A B$ & $B \star A$ & $-i$ \\
$\mathfrak{A}^{\text {cop }}$ & $B A$ & $A \star B$ & $-i$
\end{tabular}

The algebras $\mathfrak{A}$ and $\mathfrak{A}^{c o p}$ adopt one possible convention linking observables to symmetries, the Lie product $A \star B$, while $\mathfrak{A}^{o p}$ and $\mathfrak{A}^{c}$ adopt the opposite convention, the opposite Lie product $(A \star B)^{o p}=B \star A .{ }^{14}$ Furthermore, by definition any Lie product must satisfy the condition $A \star B=-(B \star A)$, so if we choose the opposite Lie product, the tangent vectors defined by (5) will point in opposite directions. The canonical anti-isomorphism and conjugate-isomorphism linking $\mathfrak{A}$ with $\mathfrak{A}^{\text {op }}$ and $\mathfrak{A}^{c}$ reverse the Lie product. They flip the generating relationship between observables and symmetries. Down the line, this will prove to be a central part of the explanation for CPT symmetry.

\subsection{Tomita-Takesaki Modular Theory}

We have gone from no suspects to an entire slew of them. Every $C^{*}$-algebra employed by AQFT comes equipped with a canonical conjugate-antiautomorphism as well as a family of related mappings connecting the algebra to its opposite, conjugate, and conjugate-opposite algebra. We do not expect every algebraic symmetry to be a symmetry, however; symmetries must preserve transition probabilities, and by Wigner's theorem, only algebraic symmetries that are implemented by unitary or antiunitary operators in a given representation will do this. Do we have reason to think that any of our suspects will always be unitarily or antiunitarily implementable?

In general the canonical involution will not be unitarily or antiunitarily implementable, but under certain technical conditions that are guaranteed to hold for every local vacuum algebra (via the Reeh-Schlieder theorem), the involution can be split into two pieces, one of which is antiunitary. Furthermore this antiunitary piece implements the canonical anti-isomorphism between $\mathfrak{A}$ and $\mathfrak{A}^{o p}$. This is our third important observation and the subject of Tomita-Takesaki modular theory.

\footnotetext{
${ }^{14}$ There will typically be infinitely many Lie products compatible with the given Jordan product. Every such compatible Lie product has a unique opposite.
} 
In its most general mathematical setting, modular theory studies the action of a von Neumann algebra, $\mathfrak{M}$, on a Hilbert space, $\mathcal{H}$, with a cyclic, separating vector, $\Phi$. The former means that $\overline{\mathfrak{M} \Phi}=\mathcal{H}$ (where the overline denotes closure in the Hilbert space norm topology), and the latter means that $A \Phi=B \Phi$ entails $A=B$. As a result, we can use $\Phi$ to translate between algebraic structure on $\mathfrak{M}$ and geometric properties of $\mathcal{H}$. In general, the canonical algebraic involution does not give rise to an isometry of the Hilbert space structure, but can always be represented as a reflection with an additional "twist."

Using $\Phi$, we can define the (generally unbounded) antilinear operator,

$$
S_{0} A \Phi=A^{*} \Phi
$$

for all $A \in \mathfrak{M}$. This can be extended to a closed, antilinear operator, $S$, defined on a dense subset of $\mathcal{H}$. Any such operator has a unique polar decomposition into a partial isometry and a positive, self-adjoint (generally unbounded) operator called the modulus. In the present case, the polar decomposition of $S$ is given by:

$$
S=J \Delta^{1 / 2},
$$

with partial isometry $J$ and modulus $\left(S^{*} S\right)^{1 / 2}=\Delta^{1 / 2}$. It can be shown that $J=J^{*}=J^{-1}$, and thus $J$, called the modular conjugation, is both antiunitary and self-adjoint. The positive operator $\Delta$ is called the modular operator. The Hilbert space action of the algebraic involution can thus be broken up into a reflection, $J$, with an additional twist, $\Delta^{1 / 2}$.

Together, the operators $\Delta, J$ have a rich structure that forms the basis of Tomita-Takesaki modular theory. Its central theorem establishes the existence of a canonical group of automorphisms of $\mathfrak{M}$ and a canonical anti-isomorphism between $\mathfrak{M}$ and its commutant $\mathfrak{M}^{\prime}$ :

Theorem (Tomita-Takesaki). If $\mathfrak{M}$ is a von Neumann algebra acting on a separable Hilbert space $\mathcal{H}$ with a cyclic and separating vector $\Phi \in \mathcal{H}$, then

$$
\begin{aligned}
& \text { (i) } J \Phi=\Phi=\Delta \Phi, \\
& \text { (ii) } \Delta^{i t} \mathfrak{M} \Delta^{-i t}=\mathfrak{M}, \forall t \in \mathbb{R}, \\
& \text { (iii) } J \mathfrak{M} J=\mathfrak{M}^{\prime}
\end{aligned}
$$

where $\Delta, J$ are the associated modular invariants. ${ }^{15}$

\footnotetext{
${ }^{15}$ In the case where $S$ is a bounded operator an elementary proof can be given. See Blackadar (2006, Thm. III.4.3.2.). The unbounded case is highly non-trivial. See Takesaki (2000, Ch. VI-VII) and Kadison and Ringrose (1997, Ch. 9.2) for different versions of the full proof.
} 
Since $\Delta$ is positive, $\Delta^{i t}$ is unitary, and (ii) defines a strongly continuous 1-parameter automorphism group of $\mathfrak{M}$ - the modular automorphism group. By (iii), the adjoint action of the modular conjugation generates an anti-isomorphism and an equivalent conjugate-isomorphism between $\mathfrak{M}$ and $\mathfrak{M}^{\prime}$. This allows us to canonically identify $\mathfrak{M}^{\prime}$ with $\mathfrak{M}^{o p}$ and $\mathfrak{M}^{c}$ :

Lemma 2. Let $\mathfrak{M}$ be a von Neumann algebra with a cyclic and separating vector $\Phi \in \mathcal{H}$, and let $\Delta, J$ be the associated modular objects. $J$ defines natural ${ }^{*}$-isomorphisms $\psi^{o p}: \mathfrak{M}^{\prime} \rightarrow \mathfrak{M}^{o p}$ and $\psi^{c}: \mathfrak{M}^{\prime} \rightarrow \mathfrak{M}^{c} .{ }^{16}$

Thus within the setting of modular theory, we find that the reflection symmetry relating $\mathfrak{M}$ and $\mathfrak{M}^{o p}$ (equiv. $\mathfrak{M}^{c}$ ) is always antiunitarily implemented, with the commutant $\mathfrak{M}^{\prime}$ identified with $\mathfrak{M}^{o p}$ (equiv. $\mathfrak{M}^{c}$ ).

In AQFT, the Reeh-Schlieder theorem guarantees these conditions hold for local algebras in the vacuum representation:

Theorem (Reeh-Schlieder). If a model of AQFT satisfies the Haag-Kastler axioms and weak additivity, then the vacuum state is cyclic and separating for every $\mathfrak{R}_{\omega}(O)$ such that $O^{\prime}$ is a proper subset of Minkowsi spacetime. ${ }^{17}$

So every local algebra in the vacuum sector has a canonical antiunitary conjugation, $J$, the reflection portion of the Tomita operator, $S$, representing the algebraic

\footnotetext{
${ }^{16}$ Proof. The mapping $j^{*}: \mathfrak{M} \rightarrow \mathfrak{M}^{\prime}$ defined $j(A):=J A^{*} J$ is the required anti-isomorphism, and the mapping $j: \mathfrak{M} \rightarrow \mathfrak{M}^{c}$ defined $j(A):=J A J$ is the equivalent conjugate isomorphism. To check this, note that $j^{*}(A B)=J(A B)^{*} J=J B^{*} A^{*} J=J B^{*} J J A^{*} J=j^{*}(B) j^{*}(A)$ and $j^{*}(i I)=J(i I)^{*} J=J(-i I) J=i I$ since $i I \in \mathfrak{M} \cap \mathfrak{M}^{\prime}$ and for any such central element $J A J=A^{*}$. Thus $j^{*}$ is an anti-isomorphism. It follows from a similar calculation that $j$ preserves products but $j(i I)=-i I$, so $j$ is a conjugate-isomorphism. (These morphisms are natural since $J$ is uniquely fixed by $\mathfrak{M}$ and $\Phi$.) Using Lemma 1 , we can then define the natural isomorphisms $\psi^{o p}:=\varphi^{o p} \circ\left(j^{*}\right)^{-1}$ and $\psi^{c}:=\varphi^{c} \circ j^{-1}$.

${ }^{17}$ This is the first instance of an analytic continuation argument in the proof of the CPT theorem. Here is the main idea: for some region $O$, suppose that $\Phi$ is orthogonal to $\mathfrak{R}_{\omega}(O) \Omega$. $\Phi$ will also be orthogonal to $U(a) \mathfrak{R}_{\omega}(\tilde{O}) U(-a) \Omega$ where $\tilde{O}$ is any subregion strictly contained in $O$ and $U(a)$ are sufficiently small translations. The spectrum condition entails that the vector-valued function $U(a) \Omega$ has analytic extension to the forward tube $T\left(V^{+}\right):=\left\{z \in \mathbb{C}^{4} \mid \operatorname{Im} z \in \overline{V^{+}}\right\}$, where $V^{+}$is the forward lightcone. This fact is used to show that the function $\left\langle\Phi, U(a) \Re_{\omega}(\tilde{O}) U(-a) \Omega\right\rangle$ is the boundary value of a holomorphic function on the forward tube that vanishes in some neighborhood of the origin and therefore vanishes everywhere. Consequently $\Phi$ is orthogonal to $U(a) \Re_{\omega}(\tilde{O}) U(-a) \Omega$ for all translations. Weak additivity then entails that $\Phi=0$, and so $\Omega$ is cyclic for $\mathfrak{R}(O)$. Microcausality entails the separating property. (For a full proof, see Horuzhy 1990, Thm. 1.3.1.) It should be noted that this result generalizes to any state analytic for the energy including DHR/BF charge states.
} 
involution. Lemma 3 then entails that the reflection symmetry linking $\mathfrak{R}_{\omega}(O)$ to $\mathfrak{R}_{\omega}(O)^{o p}$ (equivalently $\mathfrak{R}_{\omega}(O)^{c}$ ) is always antiunitarily implemented with $\mathfrak{R}_{\omega}(O)^{\prime}$ identified with $\mathfrak{R}_{\omega}(O)^{o p}$ (equivalently $\mathfrak{R}_{\omega}(O)^{c}$ ). As we will go on to see, this has important geometric ramifications since commuting algebras are associated with spacelike separated regions.

\subsection{Time Reversal}

The local modular conjugation operators are prime suspects for implementing time reversal transformations. In AQFT, the dynamics are encoded in the strongly continuous representation of the translation subgroup whose existence is posited by the covariance axiom. Relative to a given Lorentz frame, we can write the time evolution of an arbitrary observable as

$$
\alpha_{t}(X)=e^{i t P_{0}} X e^{-i t P_{0}}
$$

where $P_{0}$ is the global Hamiltonian.

Choosing an arbitrary $t=0$ allows us to identify time evolved observables in two distinct temporal directions, $t$ and $-t$. As Roberts (2017) emphasizes, in any quantum theory a time reversal transformation, $T$, should reverse the temporal ordering of observables (in the Heisenberg picture) while preserving the length of temporal intervals, thus mapping observables at time $t$ to observables at time $-t$. Moreover, it should be involutive, $T^{2}=I$. If the theory is time reversal invariant (in any generalized sense), then $T$ should also be a symmetry, hence implemented by a unitary or antiunitary operator. In addition, it should commute with $P_{0}$ so that the dynamics are unaffected by the reversal. Putting these four constraints together, we have

$$
T \alpha_{-t}(X) T=T e^{i t P_{0}} X e^{-i t P_{0}} T=e^{-i t P_{0}} T X T e^{i t P_{0}}=\alpha_{-t}(T X T) .
$$

The spectrum condition entails that the spectrum of $P_{0}$ is positive in all Lorentz frames. As a consequence, Roberts shows that the only way to consistently implement (9) with $P_{0} \neq 0$ is for $T$ to be antiunitary. The key idea is simple, but illuminating. Since the generators of the translations are unique, it follows from (9) that $T$ it $P_{0} T=-i t P_{0}$. If $T$ is unitary, then $T i t=i t T$, and so $T P_{0} T=-P_{0}$. Since unitary operators preserve inner products, if $P_{0} \neq 0$ the spectrum of $P_{0}$ cannot be bounded from below, violating the spectrum condition.

Roberts's argument sheds considerable light on why time reversal symmetries in quantum mechanics must be antiunitary. Our discussion of the dual Lie-Jordan 
product in $\S 3.2$ yields additional insight. Any time reversal symmetry worth the name must send $\alpha_{t} \mapsto \alpha_{-t}$. There are only two ways to to do this. The first is a unitary transformation sending $P_{0} \mapsto-P_{0}$. The second is an antiunitary transformation that reverses the Lie product and thus the generating relationship between $P_{0}$ and $\alpha_{t}{ }^{18}$ The first route is blocked by the spectrum condition, leaving the second as the only viable way to implement time reversal symmetry in QFT. Given the constraints linking the Lie product, $C^{*}$-product, and complex unit, antiunitary time reversal can be viewed either as a conjugate-isomorphism sending $i \mapsto-i$ with fixed $C^{*}$-product (thus sending $e^{i t} \mapsto e^{-i t}$ ) or an anti-isomorphism sending $A B \mapsto B A$ with fixed complex unit (thus sending $e^{i t} e_{-}^{-i t} \mapsto e^{-i t} e_{-} e^{i t}$ ). Since $\mathfrak{A}^{c}$ is naturally isomorphic to $\mathfrak{A}^{o p}$, the viewpoints are completely equivalent. Physically speaking, both are ways of reversing the generating relationship between observables and 1-parameter groups of symmetries.

In order to be a symmetry of AQFT, a time reversal transformation should also preserve subsystem localization information; $T$ should be a symmetry of the net of observable algebras, not just the global algebra. If $O_{1} \subset O_{2}$, and thus $\mathfrak{R}\left(O_{1}\right) \subset \mathfrak{R}\left(O_{2}\right)$, we require that $T \mathfrak{R}\left(O_{1}\right) T \subset T \mathfrak{R}\left(O_{2}\right) T$. Moreover, if a system is localized in a particular type of region (e.g., a lightcone, doublecone, spacelike wedge, spacelike cone), time reversal symmetry should preserve this localization. It should map like-regions onto like-regions.

By the main Tomita-Takesaki theorem, $J \mathfrak{R}(O) J=\mathfrak{R}(O)^{\prime}$, therefore in order for $J$ to implement a time reversal symmetry, $\mathfrak{R}(O)^{\prime}$ must be the local algebra of a region with the same geometry as $O$. In general, there is no guarantee that $\mathfrak{R}(O)^{\prime}$ will be a local algebra at all, however microcausality entails that $\mathfrak{R}\left(O^{\prime}\right) \subset \mathfrak{R}(O)^{\prime}$. If a stronger duality relation obtains, the local algebras are as large as possible consistent with microcausality and $\mathfrak{R}\left(O^{\prime}\right)=\mathfrak{R}(O)^{\prime}$. If $O$ has the same geometry as $O^{\prime}$, then the modular conjugation meets this necessary requirement.

Are there regions like this - regions that are isometric to their spacelike complement and for which we expect duality to hold quite generally? The answer is yes. The causal complement of a spacelike wedge is another spacelike wedge. In fact, spacelike wedges are essentially the only causally well-behaved regions with this property. Any open, convex, causally complete region such that $O$ and $O^{\prime}$ are isometric must be a wedge. ${ }^{19}$ Moreover, wedge duality, $\mathfrak{R}_{\omega}(W)^{\prime}=\mathfrak{R}_{\omega}\left(W^{\prime}\right)$, when

\footnotetext{
${ }^{18} \mathrm{~A}$ symmetry preserves the Lie product iff it is unitarily implemented and reverses the lie poduct iff it is antiunitarily implemented. See Alfsen and Shultz (2001, Thm. 4.27).

${ }^{19}$ Suppose some open, convex, causally complete region $O$ is isometric to $O^{\prime}$ and is not a spacelike wedge. Thomas and Wichmann (1997) prove that every closed, convex, causally, complete subset of Minkowski spacetime is the intersection of closed spacelike wedges, so $O \subset W$ for
} 
combined with the Haag-Kastler axioms is a sufficient condition for applying the tools of DHR/BF superselection theory and is expected to hold (at least in the vacuum representation) with greater generality than other forms of duality. ${ }^{20}$

\subsection{Wedge Reflection}

The focus of our investigation has narrowed to modular conjugations associated with spacelike wedges in the vacuum sector. (Incidentally, this is where most presentations of the algebraic CPT theorem begin, obscuring the physical detective work that has gotten us this far.) If wedge duality holds, then $J_{W} \Re_{\omega}(W) J_{W}=$ $\mathfrak{R}_{\omega}(W)^{\prime}=\mathfrak{R}_{\omega}\left(W^{\prime}\right)$, where $J_{W}$ is the modular conjugation associated with wedge $W$, and $W^{\prime}$ is the opposite wedge, the reflection of $W$ across one spatial direction (perpendicular to the edge of $W$ ). Thus, because of the fact that $J_{W}$ is a modular conjugation, mapping $\mathfrak{R}_{\omega}(W)$ onto its commutant, combined with duality and the unique geometry of $W, J_{W}$ is a candidate for implementing a spatial reflection. As an antiunitary involution, if $J_{W}$ commutes with the dynamics, it will also implement a time reversal symmetry.

Under the same elementary conditions needed for the Reeh-Schlieder theorem, Borchers (1992) and Weisbrock (1992) establish a remarkable result answering this latter question:

Theorem (Borchers-Weisbrock). If a model of AQFT satisfies the Haag-Kastler axioms and weak additivity, then the spectrum condition holds iff

$$
\begin{aligned}
\Delta_{W}^{i t} U(a) \Delta_{W}^{-i t} & =U\left(\Lambda_{W}(t) a\right) \\
J_{W} U(a) J_{W} & =U(r a)
\end{aligned}
$$

where $U(a)$ is the unitary representing an arbitrary translation in the vacuum representation, $\Lambda_{W}(t)$ is the unique 1-parameter group of $W$-preserving Lorentz

some wedge $W$ and thus $W^{\prime} \subset O^{\prime}$. Spacelike wedges are maximal in the lattice of open, convex, causally complete subregions, so it follows that $O^{\prime}$ is a wedge. The causal complement of a wedge is a wedge and $\left(O^{\prime}\right)^{\prime}=O$ (since $O$ is causally complete), so $O$ is a wedge, contradicting the initial assumption.

${ }^{20}$ Wedge duality entails essential duality, a technical condition needed to prove that the DHR/BF category has sufficient structure to represent charges. Essential duality requires that the dual net, $\mathfrak{R}_{\omega}(O)^{d}:=\mathfrak{R}_{\omega}\left(O^{\prime}\right)^{\prime}$, satisfies microcausality. (It should be noted that as a restriction on the family of physical states, $\{\phi\}$, the DHR/BF selection criteria can be applied whether or not duality obtains.) Another widely discussed duality condition, Haag duality, requires that $\mathfrak{R}_{\omega}(D)^{\prime}=\mathfrak{R}_{\omega}\left(D^{\prime}\right)$ for any doublecone $D$. It is equivalent to the absence of spontaneous symmetry breaking in the vacuum sector and is therefore of more limited interest although it does hold in a number of important models such as a free bosonic field. 
boosts, and $r$ is the $P_{1} T$ reflection defined by $r\left(a_{0}, a_{1}, a_{2}, a_{3}\right)=\left(-a_{0},-a_{1}, a_{2}, a_{3}\right)$, with $a_{1}$ being a spacelike translation perpendicular to the edge of the wedge. ${ }^{21}$

The modular objects $\Delta^{i t}$ and $J_{W}$ thus have the right commutation relations with the translations to be interpreted as wedge-preserving Lorentz boosts and a $\mathrm{P}_{1} \mathrm{~T}$ reflection. For 2-dimensional AQFTs, wedge duality is a direct corollary of the Borchers-Weisbrock theorem. In higher dimensions, however, counterexamples constructed by Yngvason (1994) show that things can go haywire in the directions along the edge of the wedge and the modular invariants may not map doublecones onto doublecones.

To ensure that the modular invariants act geometrically, additional analyticity assumptions are needed. Borchers (1995, 1996a, 1998, 2000) identifies two such conditions that are equivalent to wedge duality and the Bisognano-Wichmann property. Let $D$ be a doublecone contained within a spacelike wedge, $W$, and let $K(D) \subset W$ denote the cylindrical set obtained by translating $D$ in some direction parallel to the edge of the wedge. Wedge duality requires that there be enough elements $A \in \mathfrak{R}_{\omega}(K(D))$ such that $U\left(\Lambda_{W}(t)\right) A \Omega$ has bounded analytic continuation into the strip $S(-1 / 2,0)$. For any such element,

$$
U\left(\Lambda_{W}(-i / 2)\right) A \Omega=\hat{A} \Omega
$$

where $\hat{A}$ is affiliated with $\mathfrak{R}_{\omega}(K(-D)) \subset \mathfrak{R}_{\omega}\left(W^{\prime}\right)$. The Bisognano-Wichmann property requires that in addition, there is a large enough set of such analytic elements closed under involution. This motivates the following:

\footnotetext{
${ }^{21}$ Borchers originally proved the forward direction and Weisbrock the converse. The forward proof has since been greatly streamlined by Florig (1998). The key idea is as follows: spacelike translations along any direction in the characteristic 2-plane of $W$ form a positively generated 1-parameter group $U(s)$, such that $U(s) \Omega=\Omega$ and $U(s) \Re(W) U(-s) \subset \mathfrak{R}(W)$ for $s \geq 0$ (a group of so-called half-sided translations). Using the fact that $U(s)$ is positively generated, along with the analytic properties of the modular automorphism group encoded in the KMS condition, Florig shows that the function

$$
f(z)=\left\langle\Delta_{W}^{i \bar{z}} A^{\prime} \Omega, U\left(e^{2 \pi z}\right) \Delta_{W}^{-i z} A \Omega\right\rangle
$$

which is analytic in the interior of the complex strip $S(0,1 / 2)$, can be extended to a holomorphic function which is bounded, and thus constant. (Here, $A$ and $A^{\prime}$ are arbitrary elements of $\mathfrak{R}(W)$ and $\mathfrak{R}(W)^{\prime}$ respectively.) This entails, in particular, that $\Delta_{W}^{i t} U\left(e^{2 \pi t} s\right) \Delta_{W}^{-i t}=$ $\Delta_{W}^{i 0} U\left(e^{2 \pi 0} s\right) \Delta_{W}^{-i 0}=U(s)$ and $J_{W} U(-s) J_{W}=U(s)$. Extending these commutation relations to arbitrary translation vectors, $a$, is then a straightforward calculation exploiting the algebraic and analytic properties of $\Delta_{W}^{i t}$ and $J_{W}$.
} 
B-Analyticity The set of $A \in \mathfrak{R}_{\omega}(K(D))$ such that $U\left(\Lambda_{W}(t)\right) A \Omega$ has bounded analytic continuation into the strip $S(-1 / 2,0)$ is ${ }^{*}$-strong dense in $\mathfrak{R}_{\omega}(K(D))$.

B-Reality The set of $A \in \mathfrak{R}_{\omega}(K(D))$ such that both $U\left(\Lambda_{W}(t)\right) A \Omega$ and $U\left(\Lambda_{W}(t)\right) A^{*} \Omega$ can be analytically continued, with $\hat{A}^{*}=\widehat{A}^{*}$, is ${ }^{*}$-strong dense in $\mathfrak{R}_{\omega}(K(D))$.

Theorem (Borchers). If a model of AQFT satisfies the Haag-Kastler axioms and the wedge intersection property, then

(i) wedge duality holds in the vacuum representation iff B-analyticity holds,

(ii) the Bisognano-Wichmann property holds iff wedge duality and B-reality hold. ${ }^{22}$

Setting $V\left(\Lambda_{W}(t)\right):=\Delta_{W}^{i t}$, this theorem allows us to define a unitary representation of the Poincaré group, called the minimal representation, which acts covariantly on the observable net and satisfies the spectrum condition. ${ }^{23}$ Because of the analytic

${ }^{22}$ The wedge intersection property is only needed for (ii). Borchers's proof is very technical, but we can gain some understanding of it by focusing on the significance of the cylindrical sets $K(D)$ and equation (10). Note that for any $K(D) \subset W, K(D)=(W+a) \cap\left(W^{\prime}+b\right)$ where $a, b$, are spacelike translations in the characteristic 2-plane of $W$. We consider two algebras $\mathfrak{R}_{\omega}(K(D))=\mathfrak{R}_{\omega}(W+a) \cap \mathfrak{R}_{\omega}\left(W^{\prime}+b\right)$ and $\widehat{\mathfrak{R}}_{\omega}(K(D))=\mathfrak{R}_{\omega}(W+a) \cap \mathfrak{R}_{\omega}(W+b)^{\prime}$. It follows that, $\mathfrak{R}_{\omega}(K(D)) \subseteq \widehat{\Re}_{\omega}(K(D))$.

It follows from the Borchers-Weisbrock theorem that $\Delta^{i t}$ and $U\left(\Lambda_{W}(t)\right)$ commute, and since both leave $\Omega$ invariant, they differ by a 1-parameter group $F_{W}(t)$. If $A \in \mathfrak{R}_{\omega}(K(D))$ is an analytic element, then at the lower boundary of $S(-1 / 2,0)$

$$
\begin{aligned}
U\left(\Lambda_{W}(-i / 2)\right) A \Omega & =F_{W}(-i / 2) \Delta_{W}^{1 / 2} A \Omega \\
& =F_{W}(-i / 2) J_{W} A^{*} J_{W} \Omega \\
& =J_{W} F_{W}(-i / 2) A^{*} J_{W} \Omega
\end{aligned}
$$

where the second line follows from the definition of the modular invariants, $S=J \Delta^{1 / 2}$, and the last line by the Borchers-Weisbrock theorem. In general, $F_{W}(-i / 2) A^{*}$ is not a bounded operator, but it is affiliated with $\mathfrak{R}_{\omega}(K(D)) \subset \mathfrak{R}_{\omega}(W+a) \subset \mathfrak{R}_{\omega}(W)$. Thus by the TomitaTakesaki theorem and the Borchers-Weisbrock theorem, $J_{W} F_{W}(-i / 2) A^{*} J_{W}$ is affiliated with $\widehat{\mathfrak{R}}_{\omega}(K(r D)) \subset \mathfrak{R}_{\omega}(W-a)^{\prime} \subset \mathfrak{R}_{\omega}(W)^{\prime}$. Wedge duality holds iff $\widehat{\mathfrak{R}}_{\omega}(K(r D))=\mathfrak{R}_{\omega}(K(r D))$. Using Lorentz invariance, Borchers shows that this is that case iff the set of analytic elements is *-strong dense. The Bisognano-Wichmann property holds iff $F_{W}(t)$ is trivial. If so, then we have $\widehat{A^{*}} \Omega=J_{W} A J_{W} \Omega=\left(J_{W} A^{*} J_{W}\right)^{*} \Omega=\hat{A}^{*} \Omega$, and B-reality holds. The converse requires a detailed analytic continuation argument. See Borchers (2000, Thm. IV.2.2) for details.

${ }^{23}$ The key to defining the Lorentz group is to note that any Lorentz transformation is the product of boosts in three linearly independent spacelike directions, and that each such boost is 
properties of these unitaries, $\Delta_{W}^{1 / 2}=V\left(\Lambda_{W}(-i / 2)\right)$ is an element of the complex Lorentz group and hence that $J_{W}=\Delta_{W}^{1 / 2} S_{W}$ is the product of a complex Lorentz transformation and the canonical involution on the wedge algebra implemented by $S_{W}$. This allows us to show that the minimal representation contains additional reflection symmetries. In particular, we can define a "PT" operator,

$$
\Theta:=J_{W} V\left(R_{W}(\pi)\right)
$$

where $V\left(R_{W}(\pi)\right)$ implements a spatial rotation by $\pi$ in the plane along the edge of the wedge. ${ }^{24}$ The scare quotes are included to emphasize that $\Theta$ may do more than just implement a PT reflection. Indeed, in the next section we will see that it must also conjugate charge, making it a CPT operator.

But before we can proceed, one final wrinkle must be ironed out. As a consequence of the Haag-Kastler axioms and weak additivity, we have discovered that $J_{W}$ has the right algebraic properties to implement a $\mathrm{P}_{1} \mathrm{~T}$ reflection. Adding $B$-analyticity and B-reality ensures that it does in fact have such a geometric interpretation as part of an antiunitary representation of the Poincaré group. The difficulty is that this antiunitary representation may not be an extension of the original unitary representation posited by the covariance axiom. In this case we have two distinct representations, $U(\Lambda, a)$ and $V(\Lambda, a)$, encoding potentially different physics. The physics described by the minimal representation $V(\Lambda, a)$ must be $\Theta$-invariant, but there is no similar guarantee for $U(\Lambda, a)$.

Streater (1967) and Oksak and Todorov (1968) exploit this gap to construct counterexamples to the CPT and spin-statistics theorems. In all of these examples the mass spectrum is infinitely degenerate with respect to spin. As a result, such QFTs violate the split property and are thermodynamically ill-behaved, lacking

part of the stabilizer subgroup of some wedge. The translations are a bit trickier. Consider two wedges $W+a \subset W$, where $a$ is a lightlike translation in the characteristic 2-plane of W. Using the Borchers-Weisbrock theorem, it can be shown that

$$
\lim _{t \rightarrow \infty} \Delta_{W+a}^{i t} \Delta_{W}^{-i t}=\lim _{t \rightarrow \infty} V\left(\Lambda_{W+a}(t)\right) V\left(\Lambda_{W}(-t)\right)
$$

converges strongly and therefore defines a unitary operator $V(a)$ acting like a lightlike translation in the $a$ direction. The remaining translations can then be constructed as products of lightlike translations. See Borchers (2000, §IV.4) for the complete construction.

${ }^{24}$ In order to prove this it must be shown that the product $J_{W}$ maps doublecones onto doublecones and that $J_{W} V\left(R_{W}(\pi)\right)$ does not depend on the choice of a particular wedge $W$. This hinges on the analytic properties of the modular invariants, Poincaré covariance, and the special geometry of wedges. See Borchers (2000, §IV.3) for details. 
stable equilibrium states and emergent particles. Exactly how physically pathological they are remains to be investigated, but Brunetti et al. (1993) prove that if the split property obtains, even in its weaker distal form, $U(\Lambda, a)$ is the unique covariant representation of the Poincaré group acting on the vacuum representation:

Theorem (Brunetti-Guido-Longo). If a model of AQFT satisfies the Haag-Kastler axioms and the distal split property, then there can only be one covariant representation of the Poincaré group in the vacuum representation. ${ }^{25}$

So if the minimal representations exists, then the distal split property ensures that $U(\Lambda, a)=V(\Lambda, a)$ and the relevant physics is $\Theta$-invariant.

\subsection{Charge Conjugation}

The hardest part of the CPT puzzle is to understand why charge conjugation is connected to a spatiotemporal symmetry like PT. The answer lies in how the PT transformation constructed above is implemented. In effect we are performing a spatiotemporal reflection by flipping the Lie product, by changing how quantities and symmetries are linked at a fundamental level. The Lie product not only defines how spatiotemporal symmetries are tied to quantities like mass and spin, it also defines how internal symmetries are tied to gauge charges. Flipping the Lie product, while preserving the charge localization structure, maps each charge to its conjugate. This is exactly what $J_{W}$ does.

Recall from $\S 2$ that in AQFT information about global gauge symmetries is encoded in the structure of the category of localized transportable endomorphisms of the quasilocal algebra. Conjugate charges, $\varrho$ and $\bar{\varrho}$, have the defining property that $\varrho \circ \bar{\varrho}$ contains the identity. If a model of the Haag-Kastler axioms satisfies additivity and the distal split property, the statistical dimension of each charge sector is finite, and each charge has a unique conjugate up to unitary equivalence (some charges may be self-conjugate). At the heart of the algebraic CPT theorem, Guido and Longo (1992) establish the following:

\footnotetext{
${ }^{25}$ Here is the central idea: the Doplicher-Roberts reconstruction theorem (Doplicher and Roberts, 1990) shows that if the distal split property holds, the gauge group $G$ is compact and commutes with any representation of the Poincaré group. If $U(\Lambda, a)$ and $V(\Lambda, a)$ are two different representations of the Poincaré group, then the adjoint action of $U(\Lambda, a) V\left(\Lambda^{-1},-a\right)$ is an internal symmetry, and thus an element of $G$. This defines an action of the Poincaré group in $G$. Since $G$ is compact and the Poincaré group has no non-trivial finite dimensional representations, the action must be trivial and $U(\Lambda, a)=V(\Lambda, a)$.
} 
Theorem (Guido-Longo). If a model of AQFT satisfies the Haag-Kastler axioms, additivity, the distal split property, the Bisognano-Wichmann property, and the DHR/BF selection criterion, for any charge endomorphism, @, localized in a doublecone/spacelike cone

$$
\bar{\varrho}=j_{W} \circ \varrho \circ j_{W},
$$

where $j_{W}(A)=J_{W} A J_{W}$ (for all $\left.A \in \mathfrak{A}\right)$ is the endomorphism defined by the adjoint action of the vaccuum wedge modular conjugation, $J_{W}$.

Rotational invariance then entails that $\bar{\varrho}=\theta \circ \varrho \circ \theta$, where $\theta(A)=\Theta A \Theta$.

In order to understand the Guido-Longo theorem, there are two questions that must be answered - why is $j_{W} \circ \varrho \circ j_{W}$ a suitably localized transportable endomorphism, and why is it conjugate to $\varrho$ ? The answer to the first question is relatively straightforward. Since $J_{W}$ implements a $\mathrm{P}_{1} \mathrm{~T}$ reflection in the vacuum representation, it implements a $\mathrm{P}_{1} \mathrm{~T}$ reflection on the defining net of local $C^{*}$-algebras, $\{\mathfrak{A}(O)\}$, common to all sectors of the theory. ${ }^{26}$ Consequently $J_{W} \mathfrak{A}(r O) J_{W}=\mathfrak{A}(O)$, and since $J_{W}^{2}=I$, it follows that $j_{W} \circ \varrho \circ j_{W}$ is a nontrivial endomorphism on $\mathfrak{A}(r O)$ and the identity on $\mathfrak{A}\left(r O^{\prime}\right)$. Therefore $j_{W} \circ \varrho \circ j_{W}$ is localized in $r O$ a region with the same geometry at $O$. Since $\varrho$ is transportable and $J_{W}$ acts geometrically, $j_{W} \circ \varrho \circ j_{W}$ is similarly transportable.

The answer to the second question is less obvious and comes from a deep connection between conjugacy and modular inclusions. Let $\mathfrak{M}$ be an infinite factor (i.e., an infinite von Neumann algebra with a trivial center, $\mathfrak{M} \cap \mathfrak{M}^{\prime}=\mathbb{C} I$ ) acting on a separable Hilbert space with a cyclic, separating vector $\Phi$. (Eventually $\mathfrak{M}$ will be identified with $\mathfrak{R}_{\omega}(W)$.) It follows from the Tomita-Takesaki theorem that $\mathfrak{M}^{\prime}$ is also an infinite factor. Let $\varrho$ be an irreducible endomorphism of $\mathfrak{M}$ (i.e., $\left.\varrho(\mathfrak{M})^{\prime} \cap \mathfrak{M}=\mathbb{C}\right)$, and assume that $\Phi$ is also cyclic and separating for $\varrho(\mathfrak{M})$. In this setting, Longo (1984) establishs the existence of a canonical isomorphism, $\gamma_{\varrho}: \mathfrak{M} \rightarrow \varrho(\mathfrak{M})$, defined by

$$
\gamma_{\varrho}(A):=J_{\varrho} J A J J_{\varrho}
$$

where $J$ and $J_{\varrho}$ are the modular conjugations of $\mathfrak{M}$ and $\varrho(\mathfrak{M})$ with respect to $\Phi$. This canonical isomorphism in turn defines a conditional expectation, $\varepsilon_{\gamma}: \varrho(\mathfrak{M}) \rightarrow$

\footnotetext{
${ }^{26}$ Borchers and Yngvason (2000, Thm. 2.1-3) prove that if we have an algbraic CPT symmetry $\theta$ (i.e., a conjugate-linear automorphism of $\mathfrak{A}$ such that $\theta(\mathfrak{A}(O))=\mathfrak{A}(-O)$ and $\theta \circ \alpha_{a}=\alpha_{-a} \circ \theta$ for translations $\alpha_{a}$ ) then $\theta$ is represented by an antiunitary operator $\Theta$ in the universal enveloping von Neumann algebra $\mathfrak{A}^{* *}$, and that the adjoint action of $\Theta$ preserves the family of charge, particle, and thermal representations. This explains how we can lift the action of $J_{W}$ defined in the vacuum representation, to other sectors of the theory.
} 
$\mathfrak{M}$, which can be viewed as characterizing the statistical location of the subalgebra $\varrho(\mathfrak{M})$ inside $\mathfrak{M}^{27}$

If $\bar{\varrho}$ is a conjugate to $\varrho$, then by definition there exist a isometries $V, W \in \mathfrak{M}$ such that $\varrho \bar{\varrho}(A) V=V A$ and $\varrho \varrho(A) W=W A$ for all $A \in \mathfrak{M}$. Longo (1990) proves that the equation

$$
\varepsilon_{\varrho \varrho}(A):=\varrho(V)^{*} \varrho \varrho \bar{\varrho}(A) \varrho(V)
$$

also defines a conditional expectation $\varepsilon_{\varrho \varrho}: \varrho(\mathfrak{M}) \rightarrow \mathfrak{M}$. Since $\varrho$ is irreducible and $\mathfrak{M}$ is an infinite factor, there can only be one such conditional expectation due to a seminal theorem by Takesaki (1970). It follows that $\varepsilon_{\varrho \varrho}=\varepsilon_{\gamma}$, and consequently that

$$
\gamma_{\varrho}=\varrho \bar{\varrho}
$$

up to unitary equivalence. (It similarly follows that $\gamma_{\bar{\varrho}}=\bar{\varrho} \varrho$.) Putting all of these pieces together, we discover that (up to unitary equivalence) a conjugate endomorphism must have the general form

$$
\bar{\varrho}=\varrho^{-1} \circ \gamma_{\varrho}
$$

where $\varrho^{-1}$ is the inverse of $\varrho$. If $U \_U^{*}$ are unitaries implementing $\varrho$, then $\varrho^{-1}$ is the result of simply reversing the order of multiplication, $U^{*} U^{2}{ }^{28}$ The conjugate endomorphism is revealed to be something slightly more complex, the result of reversing the order of multiplication combined with a canonical isomorphism $\gamma_{\varrho}$ relating the modular structure of $\mathfrak{M}$ and $\varrho(\mathfrak{M})$. The modular conjugation of $\varrho(\mathfrak{M})$ can be written as $J_{\varrho}=\varrho(J)=U J U^{*}$. Therefore $\bar{\varrho}$ is implemented by $J U^{*} J \_J U$.

Returning now to physics, consider a transportable DHR/BF charge endomorphism $\varrho: \mathfrak{A} \rightarrow \mathfrak{A}$ localized in a doublecone or spacelike cone $O \subset W$. Wedge duality entails that $\varrho$ generates a transportable endomorphism $\varrho_{W}: \mathfrak{R}_{\omega}(W) \rightarrow \mathfrak{R}_{\omega}(W)$ localized in $W$. The wedge algebra $\mathfrak{R}_{\omega}(W)$ is an infinite factor, and by the ReehSchlieder theorem, the vacuum state is cyclic and separating for both $\mathfrak{R}_{\omega}(W)$ and $\varrho\left(\Re_{\omega}(W)\right)$. For simplicity we assume that the $\varrho_{W}$ is irreducible. ${ }^{29}$ This places us

\footnotetext{
${ }^{27}$ If $\mathfrak{N} \subset \mathfrak{M}$ are von Neumann algebras acting on a Hilbert space, a conditional expectation, $\varepsilon: \mathfrak{M} \rightarrow \mathfrak{N}$, is a positive linear mapping such that $\varepsilon(N)=N$ for all $N \in \mathfrak{N}$. Such mappings must be norm-1 projections from $\mathfrak{M}$ onto $\mathfrak{N}$ and generalize the notion of a conditional expectation from classical probability theory to a non-commutative context. If $M \in \mathfrak{M}$ and $\phi$ is a normal state on $\mathfrak{M}$ that is compatible with $\varepsilon$ (i.e., $\phi(M)=\phi(\varepsilon(M))$ ), then $\varepsilon(M)$ can be interpreted as the expectation value of $M$ conditional on $\phi(\mathfrak{N})$. In particular, $\varepsilon(M)$ reduces to a classical conditional probability for families of compatible observables.

${ }^{28}$ In the DHR/BF picture where $\varrho$ is interpreted as a localized charge creating morphism, $\varrho$ will always be unitarily implementable within its localization region, however the corresponding unitaries do not give rise to a unitary equivalence between charge sectors.

${ }^{29}$ Nothing turns on this simplification. See Guido and Longo (1992) for the general case.
} 
in the general mathematical setting discussed above.

Using equation (15), we can define a $W$-localized conjugate endomorphism $\bar{\varrho}_{W}=\varrho_{W}^{-1} \circ \gamma_{\varrho_{W}}$. Choosing unitaries $U_{-} U^{*}$ implementing $\varrho_{W}$, a straightforward calculation shows that

$$
\varrho_{W}=\left\{\begin{array}{ll}
U_{-} U^{*} & \text { on } W \\
\text { id } & \text { on } W^{\prime}
\end{array} \quad \bar{\varrho}_{W}=\left\{\begin{array}{ll}
J_{W} U^{*} J_{W} J_{W} U J_{W} & \text { on } W \\
\text { id } & \text { on } W^{\prime}
\end{array} .\right.\right.
$$

Meanwhile, $j_{W} \circ \varrho_{W} \circ j_{W}$ is localized in the opposite wedge,

$$
j_{W} \circ \varrho_{W} \circ j_{W}=\left\{\begin{array}{ll}
\operatorname{id} & \text { on } W \\
J_{W} U J_{W \_} J_{W} U^{*} J_{W} & \text { on } W^{\prime}
\end{array} .\right.
$$

It follows that $J_{W} U J_{W} \bar{\varrho}_{W} J_{W} U^{*} J_{W}=j_{W} \circ \varrho_{W} \circ j_{W}$, and since $J_{W} U J_{W}$ is unitary, $j_{W} \circ \varrho_{W} \circ j_{W}$ and $\bar{\varrho}_{W}$ are thus unitarily equivalent.

This is the situation for every $W \supset O$. If it is possible to choose a consistent family of conjugates, $\left\{\bar{\varrho}_{W_{i}}\right\}$, such that

$$
\left.\bar{\varrho}_{W_{1}}\right|_{W_{1} \cap W_{2}}=\left.\bar{\varrho}_{W_{2}}\right|_{W_{1} \cap W_{2}}
$$

for every $W_{1}, W_{2} \supset O$, then this consistent family will define an endomorphism of $\mathfrak{A}$ localized in $O$ conjugate to the original $\varrho$. (The flexibility to choose different $\bar{\varrho}_{W}$ comes from the fact that charge endomorphisms are only defined up to unitary equivalence.) Guido and Longo (1992) prove that it is possible to choose such a consistent family as a consequence of the distal split property, Poincaré covariance, and the geometric action of $J_{W}$.

Thus we find that all of the special properties of $J_{W}$ are essential for explaining why it conjugates charge. Because it implements a $\mathrm{P}_{1} \mathrm{~T}$ reflection, it preserves the regions that DHR/BF charges are localized in, and thus maps objects to objects in the relevant category of localized transportable endomorphisms. Because it is an antiunitary operator, it reverses the Lie bracket and thus the order of $C^{*}$-multiplication, inverting the endomorphism. But because it is also a modular conjugation for some spacelike wedge $W$, it also implements the canonical isomorphism $\gamma_{\varrho_{W}}$ characterizing the statistical position of $\varrho_{W}\left(\mathfrak{R}_{\omega}(W)\right) \subset \mathfrak{R}_{\omega}(W)$, and thus maps $W$-localized endomorphisms onto their conjugates given by the formula $\bar{\varrho}_{W}=\varrho_{W}^{-1} \circ \gamma_{\varrho_{W}}$. In DHR/BF representations of a Poincaré covariant QFT satisfying the distal split property, each DHR/BF localized endomorphism is generated a consistent family of such wedge-localized endomorphisms, and therefore in addition to implementing a $\mathrm{P}_{1} \mathrm{~T}$ reflection, $J_{W}$ conjugates charge. 


\subsection{Summary}

The algebraic proof of the CPT theorem shows how it is always possible, in a broad class of thermodynamically well-behaved models of AQFT, to systemically reverse the generating relationship between symmetries and observables while preserving the dynamics, spectra, and localization properties of physical systems. As a consequence of the Reeh-Schlieder theorem, for any local algebra in the vacuum sector of a generic model of AQFT, the canonical involution can be broken into two pieces, one of which is the antiunitary modular conjugation operator, J. The modular conjugation maps the relevant local algebra onto its commutant, reversing the Lie product in the process. Commuting algebras are associated with spacelike separated regions, hinting at a possible geometric interpretation, and since $J$ is antiunitary, by Wigner's theorem, it is a candidate for a physical symmetry.

For spacelike wedges, the associated modular conjugation, $J_{W}$, is in fact always a physical symmetry. Because of the spectrum condition, any generalized time reversal symmetry must be implemented by an antiunitary involution that reverses the Lie product, commutes with the dynamics, and acts uniformly on spacetime. Because $J_{W}$ is a modular conjugation operator, $J_{W} \mathfrak{R}(W) J_{W}=\mathfrak{R}(W)^{\prime}$, and if wedge duality holds, $\mathfrak{R}(W)^{\prime}=\mathfrak{R}\left(W^{\prime}\right)$, suggesting that $J_{W}$ implements a $\mathrm{P}_{1} \mathrm{~T}$ reflection. Proving that $J_{W}$ commutes with the dynamics, that wedge duality holds, and that $J_{W}$ implements a uniform geometric reflection requires a detailed technical argument exploiting analyticity properties derived from covariance, the spectrum condition, and the distal split property, as well as auxiliary assumptions B-analyticity and B-reality.

The Lie product also appears in the characterization of wedge-localized charge endomorphisms, related by the formula $\bar{\varrho}=\varrho^{-1} \circ \gamma_{\varrho}$. The Lie product encodes the relational distinction between $\varrho$ and its inverse $\varrho^{-1}$, and since $J_{W}$ reverses the Lie product, it flips this distinction. Moreover since $J_{W}$ is a modular conjugation, it preserves the statistical position of subalgebras related by charge endomorphisms (encoded by the conditional expectation associated with $\gamma_{\varrho}$ ). Because $J_{W}$ acts uniformly on spacetime, it preserves all types of localization regions, and since the theory is Poincaré covariant, endomorphisms localized in doublecones and spacelike cones can be constructed from families of compatible wedge-localized endomorphisms. Consequently, $J_{W}$ also conjugates DHR/BF charges. 


\section{Discussion}

\subsection{Explaining CPT Invariance}

The story just outlined is an example of what Bain (2016) calls a structural explanation, insofar as it appeals to "mathematical constraints on a theory's state space that are independent of the specification of the theory's dynamics" (p. 155). It should be emphasized, however, that the explanation is not a purely mathematical one. While they do take the form of mathematical conditions, the assumptions in $§ 2.1$ represent important physical constraints imposed on any causal, Lorentz invariant, thermodynamically well-behaved QFT. By connecting each stage of the proof back to the Haag-Kastler axioms, and by linking CPT reflection to a systematic reversal of the generating relationship between observables and symmetries, the aim has been to illuminate how the steps of the algebraic proof trace out important physical dependency relations present in any such QFT. ${ }^{30}$ The upshot is that the only consistent way to realize these constraints is for the theory's state space to be CPT invariant, a fact which has physical consequences for its particle spectrum as well as for scattering and decay processes. A structural explanation is only as good as our understanding of how the mathematics represents the physics, both at the level of a proof's inputs and outputs, as well as its logical structure.

Of course, we do not understand the entire story yet. B-analyticity and Breality are bootstrap assumptions. Their physical interpretation and justification is a major question mark at the heart of the algebraic proof. The role of analytic continuation arguments must be better understood, even in cases like the ReehSchlieder and Borchers-Weisbrock theorems where the starting assumptions are physically well-motivated. Whether or not additivity and the wedge intersection property can be eliminated or physically motivated remains to be seen. In addition, there are significant limitations to the scope of existing algebraic proofs. It is currently not known how to extend the DHR/BF picture to describe charges associated with local gauge symmetries, and because of the infrared problem, it cannot be applied to theories with massless particles yet. The argument also crucially relies on analyticity properties associated with symmetries of a continu-

\footnotetext{
${ }^{30}$ For our purposes it can be left open exactly sorts of things these dependency relations are. They might be nomological or meta-nomological relations, counterfactual relations, grounding or constitutive relations, or a mixture thereof. (It seems unlikely that they are causal relations, however, making the CPT theorem an important prima facie example of non-causal explanation.) At this level, the story will depend not only on further analysis of the relevant physics, but on metaphysical debates about laws, modality, and fundamentality.
} 
ous spacetime manifold, as well as on microcausality holding at arbitrarily short length-scales. Consequently, it is not clear how to generalize the algebraic argument to cover effective QFTs. ${ }^{31}$ The version of the proof outlined here makes the further assumption that spacetime is flat.

Despite these limitations, there are reasons for optimism on several fronts. Recent model-dependent results due to Morinelli (2018) suggest that the BisognanoWichmann property is logically weaker than the split property, breathing life into Haag's conjecture that it can be proven from existing, physically justified axioms. The Bisognano-Wichmann property can be formulated in any spacetime with welldefined wedge regions, and it has been used to prove the CPT theorem in curved spacetimes with a large group of global isometries (Buchholz et al., 2000). More recently, the generally covariant AQFT program initiated by Brunetti, Fredenhagen, and Verch has made substantial progress towards an axiomatic version of AQFT in arbitrary curved spacetimes (Brunetti et al., 2015). ${ }^{32}$ Combined with developments in perturbative AQFT, this program has also started to provide a provisional picture of what effective QFTs with local gauge symmetry might look like in the algebraic framework (Fredenhagen and Rejzner, 2013, 2016). Strocchi (2013) tentatively identifies the physical significance of local gauge invariance with the holding of Gauss-type conservation laws for the associated charge densities, and Kijowski and Rudolph (2003) apply the DHR picture to study the superselection structure of lattice quantum chromodynamics. Meanwhile, by studying certain equivalence classes of superselection sectors called charge classes, Buchholz and Roberts (2014) have started to clarify the complex superselection strucuture of

\footnotetext{
${ }^{31}$ Although the split property and weak additivity can be replaced by distal versions that hold at some sufficiently long length-scale without affecting the details of the proof, it is much more difficult to envision relaxing microcausality and covariance.

${ }^{32}$ Fewster (2016) applies this framework to prove a general curved spacetime version of the the spin-statistics theorem, the CPT theorem's close cousin. The proof shows that any QFT on curved spacetime that can be related to a QFT on flat spacetime by certain deformations must be obey the spin-statistics connection if the flat spacetime QFT does. This suggests that flat spacetime versions of the CPT and spin-statistics theorems might continue to carry important explanatory insight in a curved spacetime context. It also could help diffuse a potentially serious objection to the logic of the algebraic proof sketched in $\S 3$. In an arbitrary curved spacetime, it is no longer true that the causal complement of a wedge is always a wedge, and this is a necessary condition in order for $J_{W}$ to implement a $\mathrm{P}_{1} \mathrm{CT}$ reflection. Borchers has conjectured that $W^{\prime}$ is a wedge only if the background spacetime is conformally equivalent to either Minkowski or deSitter spacetime (see Hollands and Rheren 2012). If Fewster's strategy works for the CPT theorem, then in general the CPT operator will not be implemented by $J_{W}$ in curved spacetime, but will rather be defined by deformations of $J_{W}$ from a corresponding flat spacetime model.
} 
massless theories. ${ }^{33}$ Together, this work suggests that key ideas from the DHR/BF picture will apply to theories like the standard model.

Bain takes a more guarded view. Citing the current inability of AQFT to rigorously model local gauge theories, he argues that the algebraic CPT theorem does not explain why the actual laws of nature are CPT invariant: "the systems of interest; those that make contact with empirical tests, lie outside the subclass of systems for which the CPT and spin-statistics theorems provide structural explanations" (Bain, 2016, p. 157). These systems, the Yang-Mills theories that comprise the standard model, can be described using techniques from perturbative Lagrangian and S-matrix formulations of QFT, but proofs of the CPT theorem in these frameworks differ significantly from those in AQFT on Bain's telling. For example, they disagree about whether or not Lorentz invariance is necessary to prove the CPT theorem, as well as about whether CPT invariance entails the spin-statistics connection or vice versa. Consequently Bain thinks "it will be hard to make a case for a common underlying mathematical structure," shared across frameworks, that a structuralist explanation of CPT invariance can appeal to (p. 156).

While I agree that right now the algebraic CPT theorem is only a potential explanation for the CPT symmetry found in nature, I think that Bain's conclusion is overly pessimistic. In my review of Bain's book (Swanson, 2018), I argue that his presentation of the algebraic CPT theorem misinterprets the physical content of modular covariance and obscures the role of Lorentz invariance, the spectrum condition, and the split property in the physical argument at the center of the theorem. In doing so, it overemphasizes differences and underestimates commonalities between the algebraic CPT theorem and proofs couched in other frameworks. Upon closer inspection, we can identify a set of core of assumptions which appear (in slightly different forms) in virtually all known versions of the CPT theorem: restricted Lorentz invariance, energy positivity, causality, finite particle multiplicity, and analyticity.

Our detective work in $\S 3$ reinforces this critique. Unlike some versions of the

\footnotetext{
${ }^{33}$ As in the massive case, charge endomorphisms are localized in spacelike cones. Unlike the BF picture where the direction of the cone is arbitrary, however, in massless theories the direction of the cone determines an additional superselected global observable associated with the asymptotic boundary conditions of soft photon clouds. Sectors are labeled by the value of the total charge along with this asymptotic flux parameter. Although we currently lack tools to define a tensor product on the relevant category of representations, Buchholz and Roberts show that considering equivalence classes of sectors with the same global charge, many of the tools from the DHR/BF picture can be imported into this new setting.
} 
algebraic CPT theorem, the proof outlined there explicitly displays this common logical form. The covariance axiom, spectrum condition, and microcausality axiom enforce Lorentz invariance, energy positivity, and causality, while the split property ensures that the spectrum of any particle representation is at most finitely degenerate. Together, the covariance axiom, spectrum condition, and Tomita-Takesaki modular theory entail important analyticity properties (sufficient to establish the CPT theorem in 2-dimensional models), while B-analyticity and B-reality supply additional constraints needed in higher dimensions. Moreover, our analysis locates the seed of CPT reflection symmetry in a systematic reversal of the algebraic Lie product, a structure found not only in AQFT, but in every formulation of QFT that represents observables using Hilbert space operators including Lagrangian, S-matrix, and Wightman QFT. ${ }^{34}$

While there are significant structural commonalities between proofs in different frameworks, there are also non-trivial mathematical differences. Whether these stem from a deep disagreement about the fundamental character of QFT, as Bain contends, or if they are simply the product of different modeling techniques and goals, remains an open question. Regardless, a strong case can be made that the algebraic approach offers us the best explanatory insight into the CPT theorem at present. Unlike Lagrangian and Wightman QFT which start with assumptions about gauge-dependent field operators, the Haag-Kastler axioms characterize constraints on gauge-independent physical quantities, making their physical interpretation and justification more direct. Moreover, there are models of CPT invariant AQFTs that violate the Wightman axioms, that are not the quantization of any known classical Lagrangian, and which do not satisfy the assumptions of Haag-Ruelle scattering theory, a mathematically rigorous analogue of the standard S-matrix picture (Summers, 2012; Lechner, 2015). While the Wightman axioms are known to fail for QFTs with local gauge symmetry (Strocchi, 2013, ch. 6), the jury is still out on the Haag-Kastler axioms. All of this suggests that AQFT has a wider scope than other frameworks, providing a better characterization of what all relativistic QFTs have in common.

So even though we must wait for further developments in constructive AQFT to determine whether the algebraic CPT theorem gives us the right story about our own universe, we can be reasonably confident that central ideas from the proof will be part of the eventual explanation. In the interim, even as a provisional

\footnotetext{
${ }^{34}$ In some frameworks, including perturbative AQFT (Brunetti et al., 2009), the local algebras are not $C^{*}$-algebras but more general types of *-algebras. Nonetheless, such algebras contain a canonical involution operation, and thus we might hope to find a suitable generalization of the ideas sketched in $\S 3.1-2$.
} 
explanation, it can shed light on a number of conceptual puzzles about the nature of CPT symmetry including the mysterious link between temporal, spatial, and charge orientation.

\subsection{Antimatter and Time Reversal}

One of the greatest insights of the $\mathrm{DHR} / \mathrm{BF}$ analysis, is that the algebraic description of charge structure outlined in $§ 2.2$ is physically equivalent to the more traditional picture of charge structure arising from conservation laws associated with internal gauge symmetries. If the observable algebras are generated by field operators as in Lagrangian and Wightman QFT, the observable net corresponds to the gauge invariant portion of the underlying field algebra. In this context, we can characterize superselection sectors using irreducible representations of the relevant gauge group $G$. For instance, in the standard model the gauge group is $S U(3) \times S U(2) \times U(1)$. The abelian $U(1)$ piece describes the symmetries of the electromagnetic force, while the non-abelian groups $S U(2)$ and $S U(3)$ characterize the symmetries of the weak and strong force respectively. The superselected charges are defined using the Casimir invariants of the conserved currents generated by these gauge transformations. The Wightman fields act on a a single, underlying Hilbert space $H$. Under the action of $G, H$ splits into a direct sum of $G$-invariant subspaces, $H=\bigoplus H_{\sigma}$. These subspaces are in 1-1 correspondence with the superselection sectors in DHR/BF theory. Restricting the action of $G$ to $H_{\sigma}$ yields a direct sum of irreducible representations of $G$ with same character, $\sigma$. These subspaces are also $\mathfrak{A}$-invariant. Restricting $\mathfrak{A}$ to $H_{\sigma}$ yields a direct sum of quasiequivalent, irreducible representations of $\mathfrak{A}$ satisfying the DHR/BF selection criteria. Conversely, the reconstruction theorem proven by Doplicher and Roberts (1990) shows that given the category of DHR/BF representations, one can naturally reconstruct a unique minimal field algebra and gauge group, $G$.

In the field algebra picture, we find that the action of $J_{W}$ takes a given representation of $G$ to its complex conjugate representation, which models the conjugate charge sector. We do not have to look far to see why. A representation $(\pi, H)$ of $G$ can be specified by a set of generating fields, $T^{a}$, lying in the (weak closure) of the field algebra, that satisfy the commutation relations $\left[T^{a}, T^{b}\right]=i f^{a b c} T^{c}$ (where $f^{a b c}$ are the group structure constants for $G$ ). The complex conjugate representation is given by $-\left(T^{a}\right)^{*}$. Within these relations we immediately recognize the ubiquitous Lie product. The same structure which encodes how observables generate symmetries also encodes how unobservable field operators generate internal gauge transformations. Flipping this structure yields the complex conjugate representa- 
tion. It is because the same Lie structure is employed in describing both internal gauge symmetries and external spacetime symmetries that we find a connection between them.

It is worth examining just how tight this connection is. Physicists have long suspected that in quantum theories, time reversal and charge conjugation are closely linked. For example, Bell (1955) argues that proper time reversal entails charge conjugation in much the same way that it entails velocity and momentum reversal. On his view, time reversal has to be a CT transformation. Others have argued that in one way or another the matter/antimatter distinction can only be made with respect to a background temporal orientation. Feynman (1949) famously proposes a picture of antiparticles as regular particles traveling backwards in time. Wallace (2009) contends that the distinction hinges on splitting the space of solutions to the field equations into positive and negative frequency subspaces. This in turn depends on a choice of a complex structure associated with the direction of time. ${ }^{35}$

Our analysis has revealed that there is a tight connection between charge structure and temporal structure in AQFT, but it turns out to be weaker than on any of the above views. Because of the spectrum condition, any time reversal must be antiunitarily implemented, and thus reverses the Lie product. But the Lie product also helps to encode the relational distinction between different charges, and since any time reversal symmetry must act uniformly on spatial degrees of freedom, it preserves charge localization regions. Thus it appears at first glance that any time reversal operator also conjugates charge.

There are two problems with this line of thought, however. First, we have seen in $\S 3.6$ that in addition to reversing the Lie product and preserving localization regions, charge conjugation must also preserve the canonical conditional expecta-

\footnotetext{
${ }^{35}$ In the limit of no interactions, quantum electrodynamics admits an interpretation in terms of plane waves with opposite frequency. Conventionally, positive frequency solutions correspond to particle states and negative frequency solutions correspond to antiparticle states. This division into positive and negative frequency states requires a choice of complex structure on the theory's state space, essentially a choice of a Lie product, forging a link between frequency sign and translations in opposite temporal directions. Positive frequency solutions have a wavevector co-aligned with the direction of time and negative frequency solutions have an anti-aligned wavevector. Reversing the direction of time switches which wavevectors are co-aligned and antialigned.

This is the standard Lagrangian view defended by Wallace. Feynman saw a way of reading into this a story according to which annihilation reactions are just the same particle doubling back on itself in time. It simplifies certain problems, and has an elegant pictorial representation in terms of Feynman diagrams, though it is doubtful that he saw any deep metaphysical significance behind it.
} 
tion associated with $\varrho(\mathfrak{A}) \rightarrow \mathfrak{A}$. The fact that the CPT operator, $\Theta$, does so is linked to its implementation by a wedge-modular conjugation, so it is not clear that an arbitrary antiunitary time reversal operator will have this property.

Second, although it is always possible to conjugate charge by reversing the Lie product using the CPT operator, in theories where charges and and parity are treated symmetrically by the laws, it is possible to define unitary $\mathrm{C}$ and $\mathrm{P}$ operators that preserve the Lie product. In this case one can combine an antiunitary CPT reflection with unitary $\mathrm{C}$ and $\mathrm{P}$ transformations to produce a net antiunitary time reversal. This allows for theories like quantum electrodynamics which are invariant under C, P, and T symmetries independently. (More generally, it might be possible to define a unitary $\mathrm{CP}$ operator, allowing for theories invariant under $\mathrm{T}, \mathrm{CP}$, and CPT transformations.)

Thus, while $\mathrm{T}$ and $\mathrm{C}$ symmetries are closely related, they are not definitionally related like time and motion reversal. Indeed on Feynman's view it is hard to make sense of unitary charge conjugation at all. Since antiparticles just are particles traveling in the opposite temporal direction, any proper charge conjugation must involve a reverse of temporal orientation. The algebraic picture also has a distinct advantage over Wallace's view. The positive/negative frequency distinction he relies on only makes sense in the free field limit where the theory can be linearized and solutions to the equations of motion can be expanded in terms of plane waves with opposite frequency. This leads Wallace to conclude that the matter/antimatter distinction is an emergent concept which only appears in the no-interaction limit where we can coherently talk about particle states. In contrast, the algebraic picture draws a fundamental distinction between matter and antimatter which is known to apply to rigorous models of low-dimensional interacting theories like 2-dimensional Yukawa theory. After all, the CPT theorem is generally viewed as a deep, foundational result. It would be odd if it turned out merely to describe high-level, emergent phenomena. The algebraic description of charge structure reinforces this idea. If a theory is asymptotically abelian and thus has a limiting particle interpretation, one can show in the AQFT framework how matter and antimatter states are conventionally linked to opposite frequency wave solutions, thereby explaining the origins of the emergent Lagrangian picture (Mund, 2001).

\subsection{Greaves's Structural Explanation}

In her thought-provoking study of the CPT theorem, Greaves (2010) gives a different structural explanation for the physical origins of CPT symmetry. (Arntzenius 
2011 offers a similar story.) Although it has since been superseded by the more nuanced account developed by Greaves and Thomas (2014), the original, simpler version is worth investigating on its own terms first. The explanation has two main components. The first is a theory of antimatter based on Feynman's interpretation that conceptually links time reversal and charge conjugation. Particles are represented by oriented worldlines. Regular particles are co-aligned with the direction of time, while antiparticles are anti-aligned. ${ }^{36}$ As a result, any time reversal symmetry will transform particles into antiparticles and vice versa. This collapses the CPT puzzle into a PT puzzle.

The second component is a PT theorem for classical field theories in Minkowski spacetime. The CPT theorem, Greaves maintains, is essentially a relativistic phenomenon, or at least it has a direct analogue for classical field theories. The only apparent difference between her classical PT theorem and the quantum CPT theorem is the presence of antimatter which is easily handled by the Feynman picture. So according to Greaves any reasonable field theory, quantum or classical, has to be PT invariant, and if the theory has antiparticles then PT invariance is the same thing as CPT invariance.

There are two primary problems with this explanation. The first concerns the adequacy of the Feynman-inspired picture of antimatter. If particles and antiparticles are identical except for the orientation of their worldlines, then it is conceptually impossible for there to be particle/antiparticle pairs with different masses and spins. But this is a coherent possibility. Tureanu (2013) reviews a number of CPT violating QFTs with these features. Although there is considerable debate about the status of Lorentz invariance and locality in these models, they prove that particle/antiparticle mass splitting is a conceptual possibility, even if the models are not well-behaved relativistic QFTs. Unlike Greaves's picture, the algebraic view does not build this conceptual restriction into its definition of antimatter. Instead antimatter is characterized solely in terms of internal charge structure. The possibility of creation/annihilation events only requires that partners have conjugate charge, not that they are otherwise identical. This is a clear advantage of the algebraic approach, in which the validity of the CPT theorem for Lorentz invariant QFTs explains why particle/antiparticle partners in those theories have the same mass, spin, and lifetime. Indeed, from this vantage point, the CPT theorem explains why the Feynman interpretation is possible in the first place. It is because the

\footnotetext{
${ }^{36}$ The view leaves open what exactly does the orientation work. It could be a 4-momentum as in the Feynman picture. It could be a wavevector as in the standard Lagrangian picture. It could be something else entirely.
} 
theory is CPT invariant that we can interpret a forward moving antiparticle with charge $\bar{Q}$ as a backwards moving (opposite handed) regular particle with charge $Q \cdot{ }^{37}$

The second major problem concerns the scope of Greaves's explanation. Even if we accept her theory of antimatter, the classical PT theorem she relies on cannot recapture the full scope of the quantum CPT theorem. Her PT theorem considers classical field theories defined over Minkowski spacetime and has three central assumptions:

1. The fields transform as tensor quantities under diffeomorphisms of the spacetime manifold.

2. The laws have the form of partial differential equations (PDEs) which express the vanishing of some local polynomial combination of tensor fields.

3. The set of solutions is invariant under connected Poincaré transformations.

The theorem shows that any such theory must also be invariant under a discrete PT transformation.

Theorem (Greaves). In Minkowski spacetime it is impossible to directly encode only a temporal orientation using tensor fields. That is, any ploynomial combination of tensor fields that is invariant under connected Lorentz transformations is also invariant under $T$ symmetry. ${ }^{38}$

As Greaves notes, the situation is markedly different for non-relativistic field theories in Galilean spacetime, where it is possible to directly encode a temporal orientation using a special 1-form field (see Malament 2012, ch. 4.1). In this setting,

\footnotetext{
${ }^{37}$ We might attempt to salvage Greaves's view by arguing that the identification of antiparticles with anti-aligned worldlines is a physical rather than conceptual necessity in relativistic QFTs. Alternatively, we could try to accommodate particle/antiparticle mass splitting by adding additional laws of nature that forbid particles and antiparticles from having certain masses. Either way, the algebraic picture is revealed to have greater unifying power as an explanation, describing charges and antimatter in theories where these additional laws fail to hold (as well as in theories that lack a coherent fundamental notion of particle worldlines). In addition, the algebraic CPT theorem provides an explanation for when and why antiparticles can be reinterpreted as backwards moving particles. The connection between charge structure and worldline orientation lacks a clear explanation on the Greaves view, and this is only made more difficult if the connection turns out to be to be physical rather than conceptual. So while these evasive tactics are in principle available, they sacrifice much of the attractiveness of the original view.

${ }^{38}$ See Greaves (2010), fn. 12 for a proof. The same basic argument underwrites the classical PT theorem (Thm. 5.6) in Greaves and Thomas (2014).
} 
there is no analogue of the PT theorem to be found. The difference boils down to what kinds of orientation structures can be encoded by polynomial combinations of tensor fields on spacetime. In this sense, the PT theorem (and by extension the CPT theorem) is effectively a classical relativistic result.

The problem is that conditions (1) and (2) are not physically motivated. The requirement that the fields transform under a tensor representation of the Poincaré group excludes both pseudotensors and spinors. The former transform just like tensors under diffeomorphisms, but pick up a phase factor under discrete transformations. The latter are required for describing charged matter with half-integer spin, an essential ingredient in any theory like the standard model with a wide assortment of fermions in its particle zoo. Both kinds of fields can be used to construct counterexamples to the classical PT theorem, and yet both fall under the scope of the quantum CPT theorem. ${ }^{39}$

Similarly, it is difficult to see what motivates (2) apart from mathematical convenience. QFTs with non-polynomial interactions are a rich area of study in mathematical physics. Notable examples include Sine-Gordon models, Liouville field theory, and Weinberg's chiral Lagrangian for $\pi$-mesons, all of which are expected to be CPT invariant. ${ }^{40}$ In addition, in AQFT, much of the interesting theoretical work is done by axioms like the spectrum condition, microcausality, and the split property which are not PDEs at all.

With regard to these problems of scope, the algebraic account has a decisive advantage over Greaves's explanation. It expressly allows for the possibility of field operators transforming under non-tensor representations of the Poincaré group. Moreover, it is extremely liberal with the mathematical form the laws can take. Orientation structures can be naturally defined by any mathematical means whatsoever. They do not have to be encoded directly by special tensor fields. While Greaves acknowledges the limited scope of her account, she maintains that there is something puzzling about the PT connection even in the restricted class of classical tensor field theories. Presumably she thinks that the pattern of explanation

\footnotetext{
${ }^{39} \mathrm{~A}$ static PT-pseudoscalar field is a particularly simple counterexample. Spinor fields can be used to construct PT-pseudotensors as well. The bilinear currents $\bar{\psi} \gamma^{\mu} \gamma^{5} \psi$ and $\bar{\psi} \frac{i}{2}\left[\gamma^{\mu}, \gamma^{\nu}\right] \psi$ both pick up a sign under PT transformations, as does the partial derivative operator, $\partial_{\mu}$. Here $\psi$ and $\bar{\psi}$ are conjugate Dirac spinors and $\gamma^{\mu}$ are Dirac spin matrices. These pieces can be employed to construct PT violating Lagrangians.

${ }^{40}$ The Sine-Gordon equation has soliton solutions and is used in research across particle physics, condensed matter theory, and optics. Liouville field theory has applications in 2-dimensional string theory, quantum gravity, and 3-dimensional classical gravity on negatively curved spacetimes. Weinberg's Lagrangian is employed in his $S U(2) \times S U(2)$ model of $\pi$-mesons.
} 
will carry over directly to bosonic QFTs, which can be constructed without spinor fields. However, if we loosen her restrictions on laws and definability, this appears doubtful. More importantly, what makes the CPT theorem so interesting is that it holds for a broad, physically natural class of theories. The algebraic account supplies a common explanation which holds across this class. It is hard to see how Greaves's account could produce a similarly general explanation.

In newer work, Greaves and Thomas (2014) give a more complex interpretation of the Lagrangian CPT theorem drawing upon a general result dubbed strong reflection invariance. There are direct parallels between strong reflection invariance and a global reversal of the Lie product, thus the relationship between this work and the algebraic account deserves more attention than can be given here. ${ }^{41}$ The results obtained by Greaves and Thomas reinforce many of the criticisms noted above. They conclude that while there is a classical PT theorem for tensor theories, there is no similar theorem for spinor theories. In addition, the Feynman picture of antimatter no longer plays a prominent role. Instead, inspired by the standard Lagrangian picture, Greaves and Thomas characterize antimatter by a splitting of the space of field configurations into complex subspaces. Consequently their version of the CPT theorem for relativistic QFTs diverges significantly from the structural explanation developed by Greaves (2010). (They also prove a CPT theorem for relativistic classical field theories that will be discussed in §4.5.) At the same time, the restriction to polynomial interactions persists in their concept of a classical formal field theory, defined as a complex affine subspace of the set of polynomial combinations of field symbols and their derivatives, and so the algebraic CPT theorem still appears to have greater scope.

\subsection{CPT Symmetry and state space Orientation}

Although the scope of Greaves's structural account is too narrow, its central insight is promising. Even if different types of orientation structures are logically independent, there may be constraints on how laws of a particular type can encode these orientation structures. If these constraints are suitably rigid, the laws can only define one type of orientation up to a choice of the other(s). Greaves locates these constraints in laws expressing the vanishing of polynomial, Lorentz invariant tensor fields, but the story might go differently. In addition to temporal, spatial, and charge orientation, there are additional orientation structures that the

\footnotetext{
${ }^{41} \mathrm{~A}$ strong reflection is defined as a PT operation on the field symbols combined with a reversal of the order of products. As we have seen in $\S 3.2-3.3$, reversing the order of the $C^{*}$-product is one way of reversing the Lie product in QFT.
} 
laws might make use of. And in the presence of these other orientation structures, temporal, spatial, and charge orientation are less independent than it first seems.

A simple example of this phenomenon is given by the relationship between temporal, spatial, and total manifold orientations in Minkowski spacetime. Mathematically, a temporal orientation, $\tau$, can be represented by an equivalence class of co-aligned continuous timelike vector fields (Malament, 2012, Prop. 2.2.2). A spatial orientation, $\epsilon$, is a continuous labeling of right- and left-handed triads of linearly independent spacelike 4 -vectors. This can be represented by choosing a nowhere vanishing 3 -form that is orthogonal to some timelike vector field, $t^{a}$, defining a consistent notion of spatial handedness across spacetime. Any two such 3 -forms related by a strictly positive function will pick out the same orientation, thus we define $\epsilon$ as an equivalence class of 3 -forms related by strictly positive functions (Wald, 1984, p. 60, 429-434).

While both of these orientation structures require the metric, there is a third kind of orientation which depends solely on the underlying manifold structure. A total manifold orientation, $\varepsilon$, is a continuous labeling of right- and left-handed tetrads of linearly independent 4-vectors, represented by an equivalence class of nowhere vanishing 4-forms related by strictly positive functions. As we noted back in $\S 1$, specifying either a temporal or spatial orientation does not fix the other. In this sense the two structures are logically independent. If the laws employ a total manifold orientation, however, this is not the case; specifying a temporal orientation naturally defines a spatial orientation and specifying a spatial orientation naturally defines a temporal orientation. ${ }^{42}$

Although the laws of relativistic QFT do not make use of a total spacetime orientation, they do make use of a Lie product. As Alfsen and Shultz (2001, 2003) demonstrate, the Lie product can be geometrically reinterpreted as a kind of total orientation structure, not on spacetime, but on state space.

The state space of a $C^{*}$-algebra, $\mathcal{S}(\mathfrak{A})$, is the collection of all positive, normalized $\mathbb{C}$-valued linear functionals on $\mathfrak{A}$. It is a compact convex set, with extremal

\footnotetext{
${ }^{42}$ In fact any two orientation structures out of the set $\tau, \epsilon, \varepsilon$ naturally define the third. Consider $t^{a} \in \tau, e_{a b c} \in \epsilon$, and $e_{a b c d} \in \varepsilon$. First take $t^{a}$ and $e_{a b c}$. The latter must be orthogonal to some timelike vector field $s^{a}$ co-aligned with $t^{a}$. Define the 4 -form $e_{a b c d}:=-s_{[a} e_{b c d]}$. Now take $t^{a}$ and $e_{a b c d}$. Using the natural contraction operation, define the 3 -form $e_{b c d}:=t^{a} e_{a b c d}$. Since $t^{a}$ is timelike, $e_{a b c}$ must be orthogonal to some timelike vector field. Finally, take $e_{a b c}$ and $e_{a b c d}$. Define the vector field $t^{a}:=e^{a b c d} e_{b c d}$. Since $e_{a b c}$ is orthogonal to some timelike vector field, $t^{a}$ must be timelike. In all three cases since the input objects are nowhere vanishing, the defined object is similarly nowhere vanishing. Furthermore, one can show that given different choices of input objects from the same equivalence class, the defined object will be unique up to a positive function, thus the definitions are natural.
} 
points representing pure states. It has a natural order structure inherited from $\mathfrak{A}$ and its exposed faces form a lattice whose orthogonality relations mirror the spectral information encoded in the lattice of projection operators in $\mathfrak{A}$. Every selfadjoint element $A \in \mathfrak{A}_{S A}$ determines a continuous affine function $\hat{A}: \mathcal{S}(\mathfrak{A}) \rightarrow \mathbb{R}$ defined by $\hat{A}(\rho)=\rho(A)$ for all states $\rho \in \mathcal{S}(\mathfrak{A})$. Kadison (1951) proves that the mapping $A \mapsto \hat{A}$ is an isometric isomorphism between $\mathfrak{A}_{S A}$ and $\mathcal{S}(\mathfrak{A})$. Thus all of the spectral information encoded in the real Jordan algebra $\left(\mathfrak{A}_{S A}, \bullet\right)$ is encoded in the geometry of $\mathcal{S}(\mathfrak{A})$.

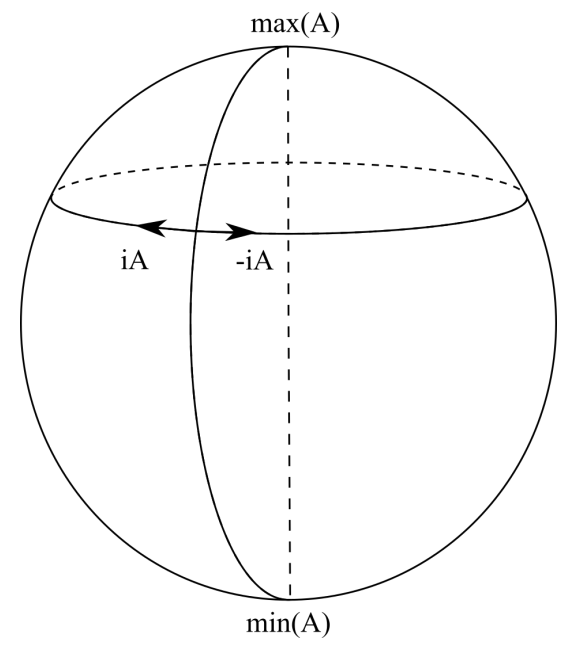

Figure 3: $\mathcal{S}\left(M_{2}\right)$ with clockwise orientation.

Alfsen et al. (1980) prove that full structure of $\mathfrak{A}$ can be recovered by equipping $\mathcal{S}(\mathfrak{A})$ with an orientation structure that determines a 1-1 pairing between observables, viewed as $\mathbb{R}$-valued affine functions on $\mathcal{S}(\mathfrak{A})$, and 1-parameter groups of symmetries of $\mathcal{S}(\mathfrak{A})$. For a 2-level quantum system, this orientation structure is easily visualized. In this case the state space, $\mathcal{S}\left(M_{2}\right)$, is isomorphic to a Euclidean 3-ball whose boundary points represent pure states and whose interior points represent mixed states. Each observable $A$ determines a bounded affine function attaining maximum and minimum values on some pair of antipodal points. The non-selfadjoint operators $i A$ and $-i A$ generate infinitesimal rotations of the 3-ball around the diameter connecting these antipodal points. There are two possible choices of orientation: $i A$ can generate clockwise and $-i A$ counterclockwise rotations, or vice versa.

This basic idea forms the basis for the general case. For an arbitrary $C^{*}$-algebra, every minimal exposed face of $\mathcal{S}(\mathfrak{A})$ is either isomorphic to a Euclidean 3-ball or 
a line segment, the former if the face is generated by distinct pure states whose GNS representations are quasiequivalent and the latter if the GNS representations are inequivalent. An orientation for $\mathcal{S}(\mathfrak{A})$ is then given by a suitably continuous choice of orientation, clockwise or counterclockwise, for each facial 3-ball. Unlike a total manifold orientation where there are only two choices, there are in general, infinitely many orientation structures of $\mathcal{S}(\mathfrak{A})$ that are in 1-1 correspondence with Jordan-compatible Lie products on $\mathfrak{A}_{S A}$ (Alfsen and Shultz, 2001, Thm. 5.73). Every such orientation, however, has a unique opposite.

This gives us additional geometric insight into the CPT operator constructed in $\S 3$. Since it reverses the Lie product, it reverses the corresponding state space orientation. It is not a product of three separate reflection symmetries, $\mathrm{C}, \mathrm{P}$, and $\mathrm{T}$, but rather a single, global reflection of QFT state space. This conclusion is reinforced by proofs of the CPT theorem in Wightman QFT and Greaves's and Thomas's recent Lagrangian proof. In both cases, a CPT operator is proven to exist without decomposing it into separate $\mathrm{C}, \mathrm{P}$, and $\mathrm{T}$ reflections. The algebraic framework, however, gives a clearer picture of the geometric origins of this operator as a systematic reversal of the generating relationship between observables and state space symmetries.

It also forms the basis for an intriguing conjecture:

Conjecture. In a causal, Lorentz invariant, thermodynamically well-behaved QFT, the choice of a state space orientation constrains the possible choices of temporal, spatial, and charge orientation structures, ensuring CPT invariance.

This has the same flavor as Greaves's original PT theorem, but with significantly different mathematical and physical content. This conjecture will be the subject of future work. ${ }^{43}$

\subsection{Classical or Quantum?}

Unlike Greaves's account, the algebraic proof of the CPT theorem employs foundational assumptions from both relativity and quantum mechanics. Covariance and microcausality appear to be essentially relativistic constraints, while the spectrum condition and modular theory are quantum mechanical in origin. This suggests that the full explanation of CPT symmetry requires ingredients from both theories. To what extent is the theorem unique to relativistic QFT?

\footnotetext{
${ }^{43} \mathrm{CPT}$, Spin-Statistics, and state space Geometry (in preparation).
} 
In a classical field theory over Minkowski spacetime all observables have determinate values at all times, this suggests that local observable algebras can be represented by commutative $C^{*}$-algebras. But in a commutative algebra, there is no natural algebraic orientation since right and left mulitplication are indistinguishable. In addition, in commutative von Neumann algebras, the modular structure becomes trivial. The modular automorphism group acts as the identity iff the generating state is tracial, i.e. $\phi(A B)=\phi(A) \phi(B)$ for all $A, B \in \mathfrak{R}$, but if the algebra is commutative, every state is a tracial state, so the modular unitaries act trivially. As a result there is no meaningful analogue of the Bisognano-Wichmann property. Furthermore, every abelian von Neumann algebra is maximal, $\mathfrak{R}=\mathfrak{R}^{\prime}$. Thus $J \mathfrak{R} J=\mathfrak{R}$, and so the modular conjugation operator cannot carry the geometric significance of a CPT operator. To compound these difficulties, Borchers (1996b, Thm. IV.6.2) shows that no net of commutative $C^{*}$-algebras can satisfy the spectrum condition.

Given the central role played by modular theory and the spectrum condition in the algebraic CPT theorem, these observations cast serious doubt on the existence of a classical theorem with similar generality and physical motivation as the quantum CPT theorem. At the same time, there are reasons to believe that this skeptical conclusion might be premature. In classical field theories, physical quantities are linked to symmetries by Noether's theorem just as in QFT. Since this generating relationship is not captured by the structure of commutative $C^{*}$ algebras, this suggests that they are not the right mathematical tools to model classical field theories. This view is adopted by the deformation quantization program, which models classical theories using dual Lie-Jordan algebras just like quantum theories. ${ }^{44}$ The principle difference between classical and quantum LieJordan algebras is the associativity or non-associativity of the underlying Jordan product. Weinstein (1997) develops the basic tools of modular theory within the setting of classical associative Lie-Jordan algebras. Whether or not such algebras can be used to evade Borchers's no-go result remains to be seen, however there are hints that the link between observables and symmetries is more tightly constrained in the quantum case than in the classical case. As Zalamea (2018) shows, since the classical Jordan product is associative, the spectral properties of classical observables are independent of their role as generators. In contrast, the non-associative quantum Jordan product directly relates the spectral properties of quantum observables to the state space symmetries that they generate. ${ }^{45}$ This suggests that

\footnotetext{
${ }^{44}$ See Landsman (1998) for an overview.

${ }^{45}$ Zalamea compares the symplectic manifold formulation of classical mechanics and the Kähler
} 
even if a classical version of the spectrum condition can be formulated, it may have very different physical consequences for the existence of reflection symmetries like CPT.

Galilean QFT offers another useful test case. Greaves attempts to explain the lack of a CPT theorem in this domain by pointing out that in Galilean spacetime (unlike Minkowski spacetime), it is possible to use tensor fields to directly encode a temporal orientation without any excess baggage. But as we have seen, this is an artificial restriction on definability. In principle though, the explanation must boil down to a difference between the structure of Galilean and Minkowski spacetime. One might hold out hope that orientation structures in Galilean spacetime are even more independent than in Minkowski spacetime. After all, the causal structure of Minkowski spacetime is encoded in a single spatiotemporal metric, while in Galilean spacetime there are two orthogonal metrics representing spatial and temporal distances separately (Malament 2012, ch. 4.1). Although it is an attractive possibility, it turns out to be false. Once we define the Galilean analogues of temporal, spatial, and total orientation, we find that any two orientation structures naturally define the third, just as in Minkowski spacetime (Weatherall, 2011).

The difference seems to come from the fact that the causal structure of Galilean spacetime is weaker than the causal structure of Minkowski spacetime. In relativistic theories, we require matter fields to propagate inside light cones, signals cannot travel faster than $c$. In contrast, Galilean spacetime has no built-in speed limit. This is reflected in the fact that the Galilean version of microcausality is a significantly weaker condition. Observables on a given simultaneity hypersurface are required to commute with each other, but this places no constraints on commutation relations at different times. Relativistic microcausality is a much more potent, 4-dimensional condition. Galilean microcausality only applies to a 3-dimensional submanifold of spacetime.

This is not the only significant difference. Unlike in the Poincaré group, in

manifold formulation of quantum mechanics in which quantum mechanical state space is viewed as a Kähler manifold, $(M, \omega, g, J)$, i.e., a symplectic manifold equipped with a compatible Riemannian metric, $g$, and almost-complex structure, $J$. In both the classical and quantum case, observables can be defined as continuous $\mathbb{R}$-valued functions that preserve all of the geometric structure of the relevant state space. In classical mechanics, the Jordan product is just the product of functions $f \bullet g=f g$. In quantum mechanics, the Jordan product is given by $f \bullet g=f g+g\left(v_{f}, v_{g}\right)$, where $g\left(v_{f}, v_{g}\right)$ is the metric-induced inner product of the Hamiltonian vector fields $v_{f}$ and $v_{g}$ generated by $f$ and $g$. On this picture, the uncertainty of a quantum observable, $\Delta f=g\left(v_{f}, v_{f}\right)$, in a given state, $\phi$, is a measure of how much the symmetry generated by $f$ changes $\phi$. 
the Galilei group temporal and spatial translations are distinct subgroups and thus have distinct generators in the corresponding Lie algebra. ${ }^{46}$ This reflects a deep sense in which time and space are separate entities in Galilean spacetime and leads to substantial modifications of the spectrum condition. In Galilean QFT, the stability of matter is secured by a mass superselection rule along with a lower bound imposed on the spectrum of a particle's internal energy, which is a completely free parameter in the theory (Lévy-Leblond, 1967). These restrictions are insufficient to endow either the modular operators or Wightman functions with the analyticity properties needed to prove the CPT theorem. ${ }^{47}$

This discussion indicates that the spectrum condition is not entirely a quantum mechanical axiom. While it ensures stability of the vacuum state, a quantum mechanical constraint, it does so by imposing restrictions on the energy-momentum operators, whose character is sensitive to the background spacetime structure. Relativistic and quantum considerations are more entangled in the axioms for AQFT than it initially appears. What is clear, however, is that the algebraic proof of the CPT theorem hinges on subtle interplay between the spectrum condition and microcausality. In Galilean QFT, the analogues of microcausality and the spectrum condition are to weak to secure the result. In classical field theories, it is not clear if we can even formulate the spectrum condition. If it turns out that we can by using associative Lie-Jordan algebras in place of commutative $C^{*}$-algebras, it is still unknown what analyticity properties will follow.

Notably, Greaves and Thomas (2014) claim to give a proof of a classical CPT theorem with the same logical structure as their version of the CPT theorem for Lagrangian QFTs. True, they are both instances of the same schema:

Theorem (Greaves-Thomas). If a classical/quantum formal field theory is (a) supercommutative and (b) invariant under a representation of the (cover of the) connected Lorentz group, then the theory is invariant under CPT reflections iff it is $\$$-hermitian. ${ }^{48}$

Supercommutativity is a version of the standard spin-statistics connection for formal field theories, while $\$$-hermiticity requires invariance under a certain involution

\footnotetext{
${ }^{46}$ The Poincare group is the semidirect product of the spatiotemporal translations with the Lorentz group, $\mathcal{P}=\mathcal{T} \rtimes \mathcal{L}$. The Galilei group is significantly more complex: $\mathcal{G}=\mathcal{U} \rtimes(\mathcal{S} \rtimes \mathcal{R})$, where $\mathcal{U}$ includes both spatial translations and Galilean boosts, $\mathcal{S}$ are the time translations, and $\mathcal{R}$ are the rotations.

${ }^{47}$ See Bain (2016, ch. 3-4) for further discussion of the failure of the CPT and spin-statistics theorems in non-relativistic spacetime.

${ }^{48}$ For proof, see Greaves and Thomas (2014, Thm. 9.6).
} 
mapping. The problem is that different notions of $\$$-hermiticity are required to prove the classical and quantum versions. In the quantum case, the relevant notion is defined with respect to the canonical involution. In the classical case, it is defined with respect to the charge conjugation involution. Thus the classical theorem asserts that if a classical field theory obeys the spin-statistics connection and is invariant under a (cover) of the Lorentz group, then it is CPT invariant iff it is $\mathrm{C}$ invariant. The quantum CPT theorem permits $\mathrm{C}$ violating theories which are nonetheless still CPT invariant. Thus there is interesting interaction between spatiotemporal and charge structure in the quantum theorem that is entirely absent in the classical version. The Greaves-Thomas classical CPT theorem is therefore markedly different from its quantum counterpart, despite initial appearances to the contrary. ${ }^{49}$

While more work is needed to fully clarify the role of relativistic and quantum mechanical assumptions in the CPT theorem, our preliminary conclusion is that, contra Greaves, the theorem essentially depends on the unification of relativity and quantum mechanics. This conclusion is broadly consistent with the historical development of QFT. Initial attempts to relativize quantum theory in the late 1920s ran aground on a cluster of problems stemming from a deep conflict between relativistic causality and energy positivity. At the time, physicists were searching for a Lorentz invariant wave equation which could play the role of the Schrödinger equation in standard quantum mechanics. The most direct strategy begins with the Schrödinger equation and replaces the the classical energy dispersion relation $E=\mathbf{p}^{2} / 2 m$ with the relativistic dispersion relation $E=\sqrt{\mathbf{p}^{2}+m^{2}}$. While the resulting equation is Lorentz invariant, it has a number of problems including the following - if we begin with a wavepacket, $\psi$, describing a particle localized in some region, under time evolution the wavepacket spreads out faster than $c$. As a result the probability of detecting the particle outside its own lightcone is nonzero. This raises the specter of faster-than-light signaling and other causality-violating paradoxes. Special relativity requires something more than just Lorentz invariance. It requires hyperbolic equations of motion describing localized initial data whose time evolution propagates with speed less than $c$.

\footnotetext{
${ }^{49}$ Flato and Raczka (1977) construct an example of a classical field theory with $\lambda \phi^{5}$ selfinteraction that is not $\mathrm{C}$ invariant. Since this is a polynomial field theory, combined with Greaves and Thomas's classical CPT theorem, this provides a prima facie example of a CPT violating classical field theory. Flato and Raczka draw a different conclusion: whether we describe this example as CPT-violating and T-symmetric or as T-violating and CPT-symmetric depends on a conventional choice of phase for the $\mathrm{T}$ operator. This shows that even in the classical case, the status of reflection symmetries is a subtle problem requiring further study.
} 
One can avoid this problem by starting with a hyperbolic wave equation like the Klein-Gordon or Dirac equation. Unfortunately, such equations typically have both positive and negative energy solutions. The presence of unbounded negative energy solutions mean that it is always possible for a system to decay into lower and lower energy states. There will be no stable matter in such a theory. Furthermore, the negative energy solutions create serious difficulties for interpreting $|\psi|^{2}$ as a probability density, destroying any hope for recovering the probabilistic formalism of standard quantum mechanics. ${ }^{50}$ One can implement a cutoff condition which throws away the negative energy states, but only at the cost of ruining hyperbolicity or Lorentz invariance. ${ }^{51}$

QFT effectively sidesteps this problem by dropping the requirement that the theory describe a finite, fixed number of particles. The negative energy states never really go away. Rather they are reinterpreted as positive energy states with opposite charge. Antiparticles and creation/annihilation processes thus emerge as critical ingredients in building a consistent QFT. But not just any old antiparticles will do the trick. They need to have exactly the same mass and spin as their conjugate partners. A number of partial results indicate that upsetting this balance will lead to violations of microcausality, Lorentz invariance, or both. ${ }^{52}$ Viewed from this angle, CPT symmetry plays a fundamental explanatory role in QFT. It is only because the theory is $\mathrm{CPT}$ invariant that we can reinterpret negative energy states as describing antiparticles in a manner consistent with the requirements of relativistic causality.

\section{Acknowledgements}

I would like to thank Jonathan Bain, David Baker, Hans Halvorson, David Malament, Bryan Roberts, David Wallace, and audiences at Pittsburgh and Princeton for comments and criticism on drafts of this paper as well as the dissertation work

\footnotetext{
${ }^{50}$ In non-relativistic quantum theory, $|\psi|^{2}$ is interpreted as a probability density, $\rho$, satisfying a continuity equation of the form $\partial \rho / \partial t+\nabla \cdot \mathbf{J}=0$. But this equation is not relativistically invariant, leading to non-conservation of the associated probability current $\mathbf{J}$. Order can be restored by replacing $\mathbf{J}$ with a relativistically invariant current, $j_{\mu}$, but now the time component, $j_{0}$, is no longer positive definite, yielding negative probabilities. (Interestingly, the Dirac equation does not suffer from this problem, and was therefore an important stepping stone towards QFT.) See Strocchi (2013), Ch. 1.2 for a discussion of this problem.

${ }^{51}$ These obstacles can be turned into a rigorous no-go theorem. See Strocchi (2013, Prop. 2.2).

${ }^{52}$ See Greenberg (2002), Peskin and Schroeder (1995), §2.4, and Weinberg (1995), §5.1, for different formulations of this kind of argument. To date, none of them have been made fully rigorous. Bain (2016, ch. 2.3) offers detailed criticism of the existing arguments.
} 
that it was based on.

\section{References}

Alfsen, E., Hanche-Olsen, H., and Shultz, F. (1980). State spaces of $C^{*}$-algebras. Acta Mathematica, 144, 267-305.

Alfsen, E. and Shultz, F. (2001). State Spaces of Operator Algebras. Basel: Birkhäuser.

Alfsen, E. and Shultz, F. (2003). Geometry of State Spaces of Operator Algebras. Basel: Birkhäuser.

Arntzenius (2011). The CPT theorem. In C. Callender (Ed.), The Oxford Handbook of Philosophy of Time. Oxford: Oxford University Press.

Bain, J. (2016). CPT Invariance and the Spin-Statistics Connection. Oxford: Oxford University Press.

Baker, D. J. and Halvorson, H. (2010). Antimatter. British Journal for the Philosophy of Science, 61, 93-121.

Bell, J. (1955). Time reversal in field theory. Proclamations of the Royal Society London, A, 231, 479-95.

Bisognano, J. and Wichmann, E. (1975). On the duality condition for a Hermitian scalar field. Journal of Mathematical Physics, 16, 985-1007.

Bisognano, J. and Wichmann, E. (1976). On the duality condition for quantum fields. Journal of Mathematical Physics, 17, 303-21.

Blackadar, B. (2006). Operator Algebras. Berlin: Springer-Verlag.

Borchers, H. J. (1992). The CPT-theorem in two-dimensional theories of local observables. Communications in Mathematical Physics, 143, 315-32.

Borchers, H. J. (1995). When does Lorentz invariance imply wedge duality? Letters in Mathematical Physics, 35, 39-60.

Borchers, H. J. (1996a). Half-sided modular inclusions and the construction of the Poincaré group. Communications in Mathematical Physics, 179, 703-23. 
Borchers, H. J. (1996b). Translation Group and Particle Representations in Quantum Field Theory. Berlin: Springer-Verlag.

Borchers, H. J. (1998). On Poincaré transformations and the modular group of the algebra associated with a wedge. Letters in Mathematical Physics, 46, 295-301.

Borchers, H. J. (2000). On revolutionizing quantum field theory with Tomita's modular theory. Journal of Mathematical Physics, 41, 3604-73.

Borchers, H. J. and Yngvason, J. (2000). On the PCT-theorem in the theory of local observables. http://arxiv.org/abs/math-ph/0012020.

Brunetti, R., Dappiaggi, C., Fredenhagen, K., and Yngvason, J. (Eds.), (2015). Advances in Algebraic Quantum Field Theory. Mathematical Physics Studies. Cham: Springer International.

Brunetti, R., Dütsch, M., and Fredenhagen, K. (2009). Perturbative algebraic quantum field theory and the renormalization group. Advances in Theoretical and Mathematical Physics, 13, 1-56.

Brunetti, R., Guido, D., and Longo, R. (1993). Modular structure and duality in conformal quantum field theory. Communications in Mathematical Physics, 156, 201-19.

Buchholz, D., Dreyer, O., Florig, M., and Summers, S. J. (2000). Geometric modular action and spacetime symmetries. Reviews in Mathematical Physics, $12,475-560$.

Buchholz, D. and Fredenhagen, K. (1982). Locality and the structure of particle states. Communications in Mathematical Physics, 84, 1-54.

Buchholz, D. and Roberts, J. E. (2014). New light on infrared problems: Sectors, statistics, symmetries and spectrum. Communications in Mathematical Physics, $330,935-72$.

Coleman, S. and Mandula, J. (1967). All possible symmetries of the S-matrix. Physics Review, 159, 1251-6.

Connes, A. (1975). A factor not anti-isomorphic to itself. Bulletin of the London Mathematical Society, 7, 171-4. 
Doplicher, S., Haag, R., and Roberts, J. E. (1969a). Fields, observables and gauge transformations I. Communications in Mathematical Physics, 13, 1-23.

Doplicher, S., Haag, R., and Roberts, J. E. (1969b). Fields, observables and gauge transformations II. Communications in Mathematical Physics, 15, 173-200.

Doplicher, S. and Roberts, J. E. (1990). Why there is a field algebra with a compact gauge group describing the superselection structure in particle physics. Communications in Mathematical Physics, 131, 51-107.

Fewster, C. (2016). On the spin-statistics connection in curved spacetime. In F. Finster, J. Kleiner, C. Röken, and J Tolksdorf (Eds.), Quantum Mathematical Physics. Basel: Birkhäuser.

Feynman, R. (1949). The theory of positrons. Physical Review, 76, 749-59.

Flato, M. and Raczka, R. (1977). On parity, charge-conjugation, and time-reversal violation in relativistic classical non-linear field theory. Letters in Mathematical Physics, 1, 443-453.

Florig, M. (1998). On Borchers' theorem. Letters in Mathematical Physics, 46, 289-93.

Fredenhagen, K. and Rejzner, K. (2013). Batalin-Vilkovisky formalism in perturbative algebraic quantum field theory. Communications in Mathematical Physics, 317, 697-725.

Fredenhagen, K. and Rejzner, K. (2016). Quantum field theory on curved spacetime: Axiomatic framework and examples. Journal of Mathematical Physics, 57, 031101-38.

Greaves, H. (2010). Towards a geometrical understanding of the CPT theorem. British Journal for the Philosophy of Science, 61, 27-50.

Greaves, H. and Thomas, T. (2014). On the CPT theorem. Studies in the History and Philosophy of Modern Physics, 45, 46-65.

Greenberg, O. (2002). CPT violation implies violation of Lorentz invariance. Physical Review Letters, 89, 231602-4.

Guido, D. and Longo, R. (1992). Relativistic invariance and charge conjugation in quantum field theory. Communications in Mathematical Physics, 148, 521-51. 
Guido, D. and Longo, R. (1995). An algebraic spin statistics theorem. Communications in Mathematical Physics, 172, 517-33.

Haag, R. (1996). Local Quantum Physics. Berlin: Springer-Verlag.

Halvorson, H. and Müger, M. (2006). Algebraic quantum field theory. In J. Butterfield and J. Earman (Eds.), Philosophy of Physics. Amsterdam: Elsevier.

Hollands, S. and Rheren, K.-H. (2012). Wedge causal manifolds - an unfinished work of Hans-Jürgen Borchers. Journal of Mathematical Physics, 53, 120401-3.

Horuzhy, S. S. (1990). Introduction to Algebraic Quantum Field Theory. Dordrecht: Kluwer Academic Publishers.

Kadison, R. V. (1951). A representation theory for commutative topological algebra. Memoirs of the American Mathematical Society, 7.

Kadison, R. V. and Ringrose, J. R. (1997). Fundamentals of the Theory of Operator Algebras, Vol. I 63 II. Providence: American Mathematical Society.

Kijowski, J. and Rudolph, G. (2003). How to define colour charge in quantum chromodynamics. In J.P. Gazeau et al. (Eds.), Group 24: Physical and Mathematical Aspects of Symmetries, Institute of Physics Conference Series No. 173.

Kuckert, B. (1997). Borchers' commutation relations and modular symmetries in quantum field theory. Letters in Mathematical Physics, 41, 307-20.

Landsman, N. (1998). Mathematical Topics Between Classical and Quantum Mechanics. New York: Springer-Verlag.

Lechner, G. (2015). Algebraic constructive quantum field theory: Integrable models and deformation techniques. In Advances in Algebraic Quantum Field Theory. Cham: Springer International

Lévy-Leblond, J.-M. (1967). Galilean quantum field theories and a ghostless Lee model. Communications in Mathematical Physics, 4, 157-76.

Longo, R. (1984). Solution of the factorial Stone-Weierstrass conjecture. Inventiones Mathematicae, 76, 145-55.

Longo, R. (1990). Index of subfactors and statistics of quantum fields II. Correspondences, braid group statistics, and Jones polynomial. Communications in Mathematical Physics, 130, 285-309. 
Malament, D. (2012). Topics in the Foundations of General Relativity and Newtonian Gravitation Theory. Chicago: Univeristy of Chicago Press.

Morinelli, V. (2018). The Bisognano-Wichmann property on nets of standard subspaces, some sufficient conditions. Annales Henri Poincaré, 19, 937-58.

Mund, J. (2001). The Bisognano-Wichmann theorem for massive theories. Annales Henri Poincaré, 2, 907-926.

Oksak, A. and Todorov, I. (1968). Invalidity of TCP-theorem for infinitecomponent fields. Communications in Mathematical Physics, 11, 125-30.

Peskin, M. and Schroeder, D. (1995). An Introduction to Quantum Field Theory. Boulder: Westview Press.

Roberts, B. (2017). Three myths about time reversal in quantum theory. Philosophy of Science, 84, 315-34.

Streater, R. F. (1967). Local fields with the wrong connection between spin and statistics. Communications in Mathematical Physics, 5, 88-96.

Strocchi, F. (2013). An Introduction to Non-Perturbative Foundations of Quantum Field Theory. Oxford: Oxford Science Publications.

Summers, S. J. (2012). A perspective on constructive quantum field theory. http://arxiv.org/abs/1203.3991.

Swanson, N. (2018). Review of Jonathan Bain's CPT Invariance and the SpinStatistics Connection. Philosophy of Science, 85, 530-9.

Swanson, N. (2014). Modular Theory and Spacetime Structure in QFT. PhD thesis, Princeton University.

Takesaki, M. (1970). Conditional expectation in von Neumann algebras. Journal of Functional Analysis, 3, 306-321.

Takesaki, M. (2000). Theory of Operator Algebras, Vol. II. Berlin: Springer-Verlag.

Thomas, L. and Wichmann, E. (1997). On the causal structure of Minkowski spacetime. Journal of Mathematical Physics, 38, 5044-86.

Tureanu, A. (2013). CPT and Lorentz invariance: Their relation and violation. Journal of Physics: Conference Series, 474, 012031-10. 
Wald, R. (1984). General Relativity. Chicago: University of Chicago Press.

Wallace, D. (2009). QFT, antimatter, and symmetry. Studies in History and Philosophy of Modern Physics, 40, 209-42.

Weatherall, J. O. (2011). The motion of a body in Newtonian theories. Journal of Mathematical Physics, 52, 32502-18.

Weinberg, S. (1995). The Quantum Theory of Fields, Vol. I. Cambridge: Cambridge University Press.

Weinstein, A. (1997). The modular automorphism group of a Poisson manifold. Journal of Geometry and Physics, 23, 379-94.

Weisbrock, H.-W. (1992). A comment on recent work of Borchers. Letters in Mathematical Physics, 25, 157-9.

Yngvason, J. (1994). A note on essential duality. Letters in Mathematical Physics, $31,127-41$.

Zalamea, F. (2018). The two-fold role of observables in classical and quantum kinematics. Foundations of Physics, 48, 1061-91. 